\title{
Dynamic Management of Portfolios with Transaction Costs under Tychastic Uncertainty
}

\author{
Jean-Pierre Aubin, Dominique Pujal, Patrick Saint-Pierre ${ }^{1}$ \\ Université Paris-Dauphine \\ Réseau de Recherche Viabilité, Jeux, Contrôle \\ http:/viab.dauphine.fr \\ September 15, 2001
}

\begin{abstract}
We use in this paper the viability/capturability approach for studying the problem of dynamic valuation and management of a portfolio with transaction costs in the framework of tychastic control systems (or dynamical games against nature) instead of stochastic control systems. Indeed, the very definition of the guaranteed valuation set can be formulated directly in terms of guaranteed viable-capture basin of a dynamical game.

Hence, we shall "compute" the guaranteed viable-capture basin and find a formula for the valuation function involving an underlying criterion, use the tangential properties of such basins for proving that the valuation function is a solution to Hamilton-Jacobi-Isaacs partial differential equations. We then derive a dynamical feedback providing an adjustment law regulating the evolution of the portfolios obeying viability constraints until it achieves the given objective in finite time. We shall show that the Pujal 85 Saint-Pierre viability/capturability algorithm applied to this specific case provides both the valuation function and the associated portfolios.
\end{abstract}

Acknowledgments The authors thank Giuseppe Da Prato, Francine Catté, Halim Doss, Hélène Frankowska, Georges Haddad, Nisard Touzi and Jerzy Zabczyk for many useful discussions and Michèle Breton and Georges Zaccour for inviting us in June 2004 to present these results in the Montréal's GERAD (Groupe d'études et de recherche en analyse des décisions).

Outline The first section is an introduction stating the problem and describing the main results presented. It is intended to readers who are not interested in the mathematical technicalities of the viability approach to financial dynamic valuation and management problems. The second section outlines the viability/capturability strategy and provides the minimal definitions and results of viability theory for deriving in the third and last section sketches the proofs of the main results

\footnotetext{
${ }^{1}$ Work supported in part by the European Community's Human Potential Programme under contract HPRN-CT2002-00281, [Evolution Equations]
} 


\section{Introduction and Survey of the Main Results}

\subsection{Statement of the Problems}

We shall describe the main results of this paper in the framework of the dynamic valuation and management of a portfolio replicating European, American options or Kairotic options (options exercised at the first instant when the value of the portfolio is above the contingent value), postponing the general case in the next section.

Let $n+1$ financial assets $i=0,1, \ldots, n$, the first one being non-risky (a bond) and the $n$ other ones being risky assets (stocks).

The components of the variable $x:=\left(x_{0}, x_{1}, \ldots, x_{n}\right) \in \mathbf{R}^{n+1}$ are the price of the non risky asset (bond), labelled $i=0$, and the prices of the $n$ risky assets (stocks), labelled $i=1, \ldots, n$, usually denoted by $S:=\left(S_{0}, S_{1}, \ldots, S_{n}\right)$ in the financial literature. A portfolio is an element

$$
p:=\left(p_{0}, p_{1}, \ldots, p_{n}\right) \in \mathbf{R}^{n+1}
$$

describing the number of shares of assets $i=0,1, \ldots, n$. The associated capital (or the value of the portfolio) $y$, usually denoted by $W$ in the financial literature ${ }^{2}$ can be written

$$
y:=\langle p, x\rangle=\sum_{i=0}^{n} p_{i} x_{i}
$$

Let assume that

1. the uncertain evolution of prices $x(t) \in \mathbf{R}^{n+1}$ of financial assets is known, rather, forecasted,

2. constraints

(a) $p(t) \in D(t, x(t))$ on the portfolios $p(t)$,

(b) $p^{\prime}(t) \in P(t, x(t), p(t))$ on the velocities $p^{\prime}(t)$ of portfolios $p(t) \in \mathbf{R}^{n+1}$ (describing transaction costs, for instance)

are given (constraints of the portfolio can be integrated in the constraints on their velocities by setting $P(t, x, p)=\emptyset$ whenever $p \notin D(t, x))$.

Let us consider a given time-independent function $\mathbf{u}: \mathbf{R}^{n+1} \mapsto \mathbf{R} \cup\{+\infty\}$, called the contingent claim, and an exercise time $T$. The general problem of dynamic portfolio management is to find an evolution law of the portfolio $p(\cdot)$ such that, whatever the uncertain evolution of the prices, one of the

\footnotetext{
${ }^{2}$ In several financial studies, a policy (of capital allocation) is an element $\pi:=\left(\pi_{0}, \pi_{1}, \ldots, \pi_{n}\right)$ where the component $\pi_{i}$ defined by $p_{i} x_{i}=\pi_{i}\langle p, x\rangle$ denotes the proportion of the capital allocated to asset $i$ are used as variables. They are defined naturally only when the capital $y:=\langle p, x\rangle$ is strictly positive. We shall not use this definition because imposing constraints on the actual number of shares of assets seems to us more realistic than imposing constraints on the proportions of shares.
} 
following option rules ${ }^{3}$ is satisfied: We require that at the exercise time $T$, the option is exercised

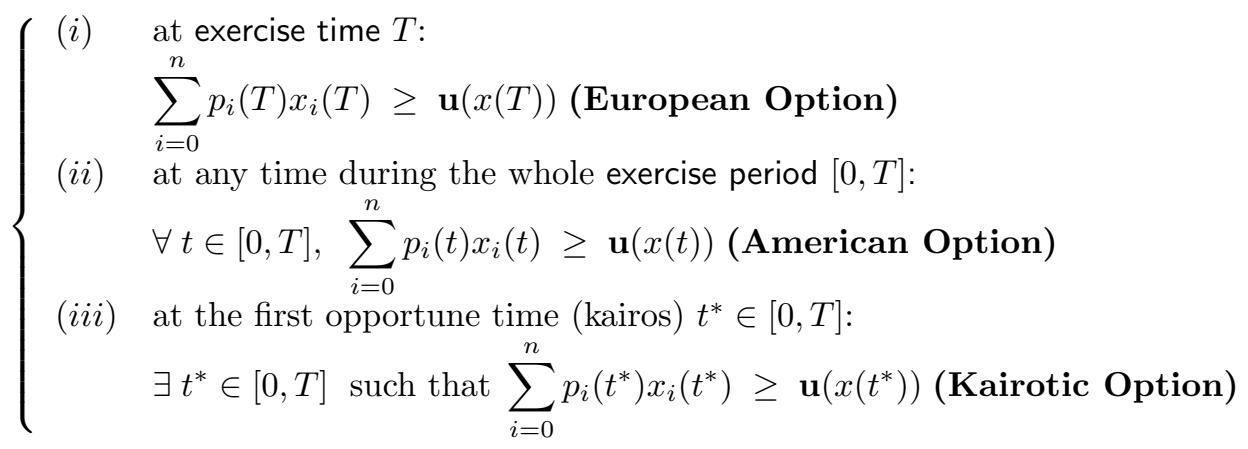

Our ultimate goal is to construct

1. the valuation function $(T, x, p) \mapsto V^{\sharp}(T, x, p) \in \mathbb{R} \cup\{+\infty\}$ associating with the exercise time $T$, the initial price $x$ of the shares and the initial portfolio the smallest initial capital $V^{\sharp}(T, x, p)$ guaranteeing that one of the above rules is satisfied whatever the uncertainty

2. and above all, the regulation map $\Gamma: \mathbb{R}_{+} \times \mathbb{R}^{n+1} \times \mathbb{R}^{n+1} \leadsto \mathbb{R}^{n+1}$ providing the a posteriori adjustment law

$$
p^{\prime}(t) \in \Gamma(T-t, x(t), p(t))
$$

governing the evolution of the portfolio necessary to solve the given management problem whatever the uncertainty.

We shall answer these questions by solving a whole family of rules containing as particular cases the three (1), use the "epigraphical approach" to transform the inequalities involves in the above rules as a viability/capturability problem and then, use some results of viability theory not only for theoretical results, but also to provide algorithms providing the valuation function and the regulation map, bypassing the numerical computation of solutions to analogues of Black and Scholes partial differential equations.

\subsection{Underlying Viability/Capturability Problem}

Indeed, in order to treat the three rules (1) as particular cases of a more general framework, we introduce two nonnegative extended functions (fonctions taking infinite values)b (constraint function) and $\mathbf{c}$ (objective function) satisfying

$$
\forall(t, x, p) \in \mathbf{R}_{+} \times \mathbf{R}_{+}^{n+1} \times \mathbf{R}^{n+1}, \quad 0 \leq \mathbf{b}(t, x, p) \leq \mathbf{c}(t, x, p) \leq+\infty
$$

and

$$
\forall p \notin D(x), \quad \mathbf{b}(t, x, p)=+\infty
$$

For example, we can associate with the initial function $\mathbf{u}$ the extended function $\mathbf{u}_{\infty}$ defined by

$$
\mathbf{u}_{\infty}(t, x, p):=\left\{\begin{array}{cc}
\mathbf{u}(x, p) & \text { if } \\
+\infty & \text { if not }
\end{array}\right.
$$

\footnotetext{
${ }^{3}$ These rules are applied in finance theory for determining the portfolios replicating options when the function $\mathbf{u}$ is regarded as a claim function.
} 
and introduce the extended function $\mathbf{0}$ defined by

$$
\mathbf{0}(t, x, p)=\left\{\begin{array}{ll}
0 & \text { if } \\
+\infty & \text { if not }
\end{array} \quad t \geq 0,\right.
$$

We replace the requirements (1) by the family of rules

$$
\begin{cases}\text { (i) } \quad \forall t \in\left[0, t^{*}\right], y(t) \geq \mathbf{b}(T-t, x(t), p(t)) \\ \\ \text { (dynamical constraint) } \\ \text { (ii) } y\left(t^{*}\right) \geq \mathbf{c}\left(T-t^{*}, x\left(t^{*}\right), p\left(t^{*}\right)\right) \\ \\ \text { (final objective) }\end{cases}
$$

associated with pairs of extended functions $(\mathbf{b}, \mathbf{c})$.

Since extended functions can take infinite values, we are able to acclimate many examples. In particular, the three rules (1) associated with a same function $\mathbf{u}: \mathbf{R}^{n+1} \times \mathbf{R}^{n+1} \mapsto \mathbf{R} \cup\{+\infty\}$ can be written in the form (3) by adequate choices of pairs $(\mathbf{b}, \mathbf{c})$ of functions associated with $\mathbf{u}$ :

$$
\begin{cases}\text { (i) } \quad & \sum_{i=0}^{n} p_{i}(T) x_{i}(T) \geq \mathbf{u}(x(T)) \text { (European Option), } \\ & \text { by taking } \mathbf{b}(t, x, p):=\mathbf{0}(t, x, p) \text { and } \mathbf{c}(t, x, p)=\mathbf{u}_{\infty}(t, x, p) \\ (\text { ii }) \quad & \forall t \in[0, T], \sum_{i=0}^{n} p_{i}(t) x_{i}(t) \geq \mathbf{u}(x(t)) \text { (American Option), } \\ & \text { by taking } \mathbf{b}(t, x, p):=\mathbf{u}(x, p) \text { and } \mathbf{c}(t, x, p):=\mathbf{u}_{\infty}(t, x, p) \\ \text { (iii) } \quad \exists t^{*} \in[0, T] \text { such that } \sum_{i=0}^{n} p_{i}\left(t^{*}\right) x_{i}\left(t^{*}\right) \geq \mathbf{u}\left(x\left(t^{*}\right)\right) \text { (Kairotic Option), } & \\ & \text { by taking } \mathbf{b}(t, x, p):=\mathbf{0}(t, x, p) \text { and } \mathbf{c}(t, x, p)=\mathbf{u}(x, p)\end{cases}
$$

The case of prescribed time is obtained in the following way:

Lemma 1.1 Problems with prescribed final time (as in portfolios replicating European and American options) are obtained with objective functions satisfying the condition

$$
\forall t>0, \mathbf{c}(t, x, p):=+\infty
$$

In this case, $t^{*}=T$ and condition (3) boils down to

$$
\begin{cases}(\text { i }) & \forall t \in[0, T], y(t) \geq b(T-t, x(t), p(t)) \\ (\text { ii }) & y(T) \geq c(0, x(T), p(T))\end{cases}
$$

Proof - Indeed, since $y\left(t^{*}\right)$ is finite and since $\mathbf{c}\left(T-t^{*}, x\left(t^{*}\right), p\left(t^{*}\right)\right)$ is infinite whenever $T-t^{*}>0$, we infer from inequality [3](ii) that $T-t^{*}$ must be equal to 0 .

Definition 1.2 The epigraph of an extended function $\mathbf{v}: \mathbb{R}_{+} \times X \times X \mapsto \mathbb{R}_{+} \cup\{+\infty\}$ is defined by

$$
\mathcal{E} p(\mathbf{v}):=\{(x, \lambda) \in X \times \mathbf{R} \mid \mathbf{v}(x) \leq \lambda\}
$$



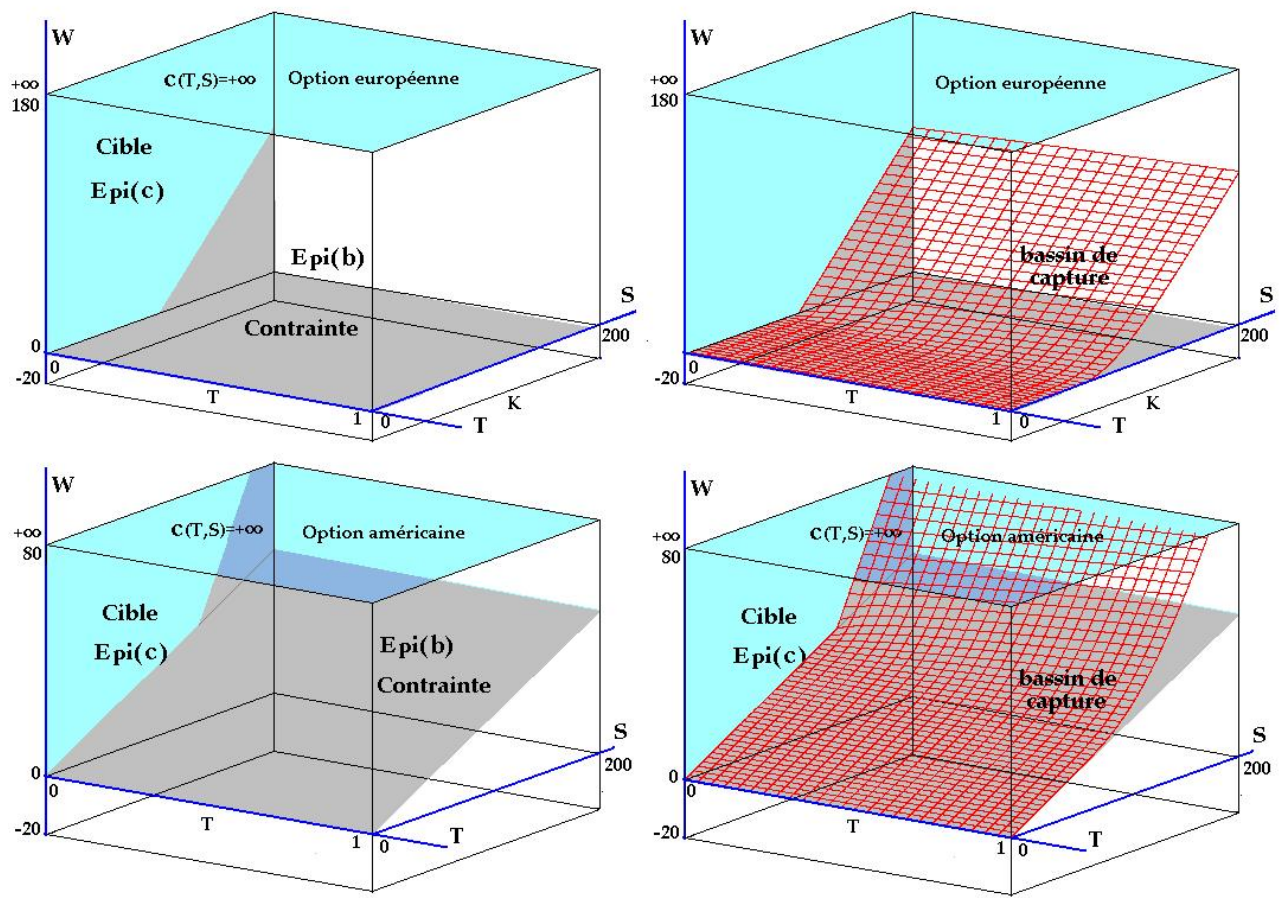

Figure 1: Epigraphs of Valuation Functions of portfolios are capture basin of a target (epigraph of function) viable in a constrained set (epigraph of another functionc: $\mathbb{R}_{+} \times X \times X \mapsto \mathbb{R}_{+} \cup\{+\infty\}$ ) viable in a constrained set (epigraph of another function $b: \mathbb{R}_{+} \times X \times X \mapsto \mathbb{R}_{+} \cup\{+\infty\}$ ) satisfying $\forall(t, x) \in \mathbf{R}_{+} \times X, \quad 0 \leq \mathbf{b}(t, x) \leq \mathbf{c}(t, x) \leq+\infty$ This illustrated here in the case of European and American Options without transaction costs.

We recall that an extended function $\mathbf{v}$ is convex (resp. positively homogeneous) if and only if its epigraph is convex (resp. a cone) and that the epigraph of $\mathbf{v}$ is closed if and only if $\mathbf{v}$ is lower semicontinuous:

$$
\forall x \in X, \quad \mathbf{v}(x)=\liminf _{y \rightarrow x} \mathbf{v}(y)
$$

We can translate the viability/capturability conditions (3) in the following geometric form:

Lemma 1.3 An evolution $t \mapsto(T-t, x(t), p(t))$ satisfies viability/capturability conditions (3) if and only if it is viable in the epigraph $\mathcal{E} p(\mathbf{b})$ until it captures the target $\mathcal{E} p(\mathbf{c})$ : This means that there exists a finite time $t^{\star} \geq 0$ such that

$$
\begin{cases}(i) & \left(T-t^{\star}, x\left(t^{\star}\right), p\left(t^{\star}\right), y\left(t^{\star}\right)\right) \in \mathcal{E} p(\mathbf{c}) \\ (i i) \quad \forall t \in\left[0, t^{\star}\right], & (T-t, x(t), p(t), y(t)) \in \mathcal{E} p(\mathbf{b})\end{cases}
$$

The reformulation of the rules (3) describing the nature of the financial problem in this framework allows us to use viability theory: In a nutshell, we shall prove that once the dynamics governing the 
evolutions of prices and portfolios are given, the epigraphs of the "valuation function" of the portfolio is the "capture basin" of the target (epigraph of a function $c: \mathbb{R}_{+} \times X \times X \mapsto \mathbb{R}_{+} \cup\{+\infty\}$ ) viable in an $=$ constrained environment (epigraph of another function $b: \mathbb{R}_{+} \times X \times X \mapsto \mathbb{R}_{+} \cup\{+\infty\}$ ) satisfying

$$
\forall(t, x) \in \mathbf{R}_{+} \times X, \quad 0 \leq \mathbf{b}(t, x) \leq \mathbf{c}(t, x) \leq+\infty
$$

The terms "valuation function" and "capture basin" will be defined in due time. The "epigraphical approach" consisting in using the properties of the epigraphs of a function was proposed by J.-J. Moreau and R.T. Rockafellar in convex analysis in the early 1960's 4 , and has been used in optimal control by H. Frankowska in a series of papers [46, 47, 49, Frankowska] and [17, Aubin \& Frankowska] for studying the value function of optimal control problems and characterize it as generalized solution (episolutions and/or viscosity solutions) of (first-order) Hamilton-Jacobi-Bellman equations, in [1, 10, [2, 3, Aubin] for characterizing and constructing Lyapunov functions, in [36, 37, 38, Cardaliaguet] for characterizing the minimal time function, in [61, Pujal] in finance and other authors since. This is this approach that we adopt and adapt here, since the concepts of "capturability of a target" and of "viability" of a constrained set allows us to study this problem under a new light (see for instance [3, Aubin] and 4, Aubin] for economic applications) for studying the evolution of the state of a tychastic control system subjected to viability constraints in control theory and in tychastic control systems or dynamical games against nature or robust control (see [63, Quincampoix], 36, 37, 38, Cardaliaguet], [39, Cardaliaguet, Quincampoix \& Saint-Pierre]. Numerical algorithms for finding viability kernels have been designed in [66, Saint-Pierre] and adapted to our type of problems in [61, Pujal] and 62, Pujal \& Saint-Pierre]).

\subsection{The Dynamics}

1. Dynamics of Prices As in papers [28, 29, 30, Bernhard], 61, Pujal] and [62, Pujal \& SaintPierre] that appeared simultaneously and independently at the end of the year 2000, as well as earlier contributions [60, Olsder], 65, Runggaldier], the evolution of the prices is governed by

$$
\forall i=1, \ldots, n, \quad x_{i}^{\prime}(t)=x_{i}(t) \rho_{i}(x(t), v(t)) \text { where } v(t) \in Q(t, x(t), p(t)) \subset \mathbb{R}^{m}
$$

where the set-valued map $Q: \mathbb{R}_{+} \times \mathbb{R}^{n+1} \times \mathbb{R}^{n+1} \leadsto \mathbb{R}^{m}$, the tychastic map associating with any triple $(t, x, p)$ a set of elements $v \in Q(t, x, p)$ regarded as tyches (or perturbations, disturbances) one of the Greek words encapsulating the concept of chance, personified by the Goddess Tyche. The size of the tychastic map represents a kind of "tychastic volatility", called "versatility".

The tychastic map (that could be a fuzzy subset, as it is advocated in [14, Aubin \& Dordan]) provides an alternative mathematical translation of evolution under uncertainty parallel to the usual mathematical translation by a diffusion in the framework of stochastic differential equations. The size of the subsets $Q(t, x, p)$ captures mathematically the concept of "tychastic versatility" - instead of "stochastic volatility": The larger the subsets $Q(t, x, p)$, the more "tychastic" the system.

Controlling a system for solving a problem (such as viability, capturability, intertemporal optimality) whatever the perturbation is the branch of dynamical games (dynamical games against nature) known among control specialists as "robust control", that we propose to call "tychastic

\footnotetext{
${ }^{4}$ see for instance [16, Aubin \& Frankowska] and [64, Rockafellar \& Wets] among many other references.
} 


Charles (Sanders) Peirce (1839-1914) introduced the concept of ty-
chastic evolution in a paper published in 1893 under the title evolu-
tionary love.
"Three modes of evolution have thus been brought before us: evolution
by fortuitous variation, evolution by mechanical necessity, and evolution by
creative love. We may term them tychastic evolution, or tychasm, anan-
castic evolution, or anancasm, and agapastic evolution, or agapasm. In
this paper, Peirce associates the concept of anancastic evolution with
the Greek concept of necessity, ananke, anticipating the "chance and
necessity" framework that motivated viability theory. Peirce was a
logician and a prolific and profound philosopher interested in evolu-
tion theory after Charles Darwin and Herbert Spencer (1820-1903),
introduced many concepts, such as abduction ("process of thought ca-
pable of producing no conclusion more definite than a conjecture")
and semiotics (the "general science of the nature of signs").

State-dependent uncertainty can also be translated mathematically by parameters on
which actors, agents, decision makers, etc. have no controls. These parameters are of-
ten perturbations, disturbances (as in "robust control" or "differential games against
nature") or more generally, tyches (meaning "chance" in classical Greek, from the
Goddess Tyche) ranging over a state-dependent tychastic map. They could be called
"random variables" if this vocabulary were not already confiscated by probabilists.
This is why we borrow the term of tychastic evolution to Charles Peirce who intro-
duced it in a paper published in 1893 under the title evolutionary love. One can
prove that stochastic viability is a (very) particular case of tychastic viability. The
size of the tychastic map captures mathematically the concept of "versatility (tychastic
volatility)" - instead of "(stochastic) volatility": The larger the graph of the tychastic
map, the more "versatile" the system.


control" in contrast to "stochastic control", where such properties must be satisfied "almost surely".

However, these apparently different two choices sharing a same philosophy can be reconciled, since there is a deep link between tychastic and stochastic problems for viability/capturability issues. Indeed, thanks to the equivalence formulas between Itô and Stratonovitch stochastic integrals and to the Strook \& Varadhan "Support Theorem" (see for instance [44, Doss]), and under convenient assumptions, stochastic viability problems are equivalent to invariance problems for tychastic systems (see [15, Aubin \& Doss]). By the way, this is in this framework of invariance under tychastic systems associated with stochastic problems that stochastic invariance issues in mathematical finance are studied ([31, Björk], [45, Filipovic], [54, Jachimiak], [57, 58, 59, Milian], [72, Tessitore \& Zabczyk], [7, 76, Zabczyk],etc.) and the annex at the end of this paper.

2. Dynamics of Portfolios From the view point of a manager, a strategy or a decision can be regarded as the velocity $p^{\prime}(t)$ of his/her portfolio, describing how and how fast he/she modifies the portfolio.

We assume that the available velocities $p^{\prime}(t)=u(t)$ of the portfolios, chosen as control $u(t)$, range over a subset $P(t, x(t), p(t))$, where the set-valued map $P: \mathbb{R}_{+} \times \mathbb{R}^{n+1} \times \mathbb{R}^{n+1} \sim \mathbb{R}^{m}$ is regarded as a cybernetic man ${ }^{5}$. Observe that this map encapsulates time/price-dependent constraints on portfolios translated by the condition $p(t) \in D(t, x(t))$ where

$$
D(t, x):=\{p \text { such that } P(t, x, p) \neq \emptyset\}
$$

For instance, transaction costs, balancing the variations resulting from the buy/sell operations on the bond and on the risky asset, can be taken into account in the definition of the set-valued map $P$, that can take the form

$$
P(t, x, p):=\left\{\begin{array}{cl}
\left\{u \in \mathbf{R}^{n+1}|| u_{i} \mid \leq \gamma_{i} \& \sum_{\emptyset=1}^{n}\left(u_{i} x_{i}+\delta_{i}\left(\left|u_{i}\right|, x_{i}\right)\right)=0\right\} & \text { if } p \in D(x) \\
\text { if not } & \text { if }
\end{array}\right.
$$

where the functions $\delta_{i}$ are the transaction cost functions and bounds $\gamma_{i}$ on the rates of exchanges of shares describe some inertia in the transaction. Other behavioral constraints can be taken into account in the cybernetic map.

The self-financing assumption meaning that it is forbidden to borrow external income to modify the capital of the portfolio, i.e., that the capital of portfolio can change only by financing it with revenues obtained by selling and buying assets (case when $\delta_{i}=0$ and $\gamma_{i}=\infty$ ) is described by

$$
P(t, x, p)=\left\{u \in \mathbf{R}^{n+1} \mid\langle u, x\rangle=0\right\}
$$

In this case, there is no constraint on the norm of the velocities of the prices, that can be as large as wanted, as long as $\langle u, x\rangle=0$, and the influence of the velocity $p^{\prime}(t)$ disappears from

\footnotetext{
${ }^{5}$ In tychastic control problems, we have two kinds of uncertainties, one described by the set-valued map $Q$, describing tychastic uncertainty, and the one described by the set-valued map $P$, encapsulating the concept of cybernetic uncertainty, providing a set of available regulation parameters (regulons), the size of it describing the "redundancy" of the cybernetic map. The larger the cybernetic map $P$, the more redundant is the system to find a regulation regulon or a control to satisfy a given property whatever the perturbation in $Q$. In some sense, the "cybernetic map" $P$ is an antidote to cure the negative effects of unknown tyches provided by the tychastic map $Q$. Redundancy compensates for versatility ... or plain ignorance
} 
the dynamics of the capital. We are in the simpler case case studied by [61, Pujal], 62, Pujal \& Saint-Pierre].

3. Dynamics of Capital Knowing the tychastic evolution of prices and the contingent evolution of portfolios describing the behavioral rule of the investor, we deduce the evolution of the capital:

$$
\left\{\begin{array}{l}
y^{\prime}(t)=\left\langle p(t), x^{\prime}(t)\right\rangle+\left\langle p^{\prime}(t), x(t)\right\rangle \\
=\sum_{i=0}^{n} p_{i}(t) x_{i}(t) \rho_{i}(x(t), v(t))+\langle u(t), x(t)\rangle \\
=y(t) \rho_{0}(x(t))-\sum_{i=1}^{n} p_{i}(t) x_{i}(t)\left(\rho_{0}\left(x_{0}(t)\right)-\rho_{i}(x(t), v(t))\right)+\langle u(t), x(t)\rangle
\end{array}\right.
$$

\subsection{Dynamic Management and Valuation of the Portfolio}

We choose any one of the rule (1), or, more generally, (3). Our ultimate goal is to construct the the valuation function $(T, x, p) \mapsto V^{\sharp}(T, x, p) \in \mathbb{R} \cup\{+\infty\}$ and above all, the regulation map $\Gamma: \mathbb{R}_{+} \times \mathbb{R}^{n+1} \times \mathbb{R}^{n+1} \leadsto \mathbb{R}^{n+1}$ and differential inclusion

$$
p^{\prime}(t) \in \Gamma(T-t, x(t), p(t))
$$

governing the evolution of the portfolio under tychastic uncertianty:

1. The Valuation Function $(T, x, p) \mapsto V^{\sharp}(T, x, p) \in \mathbb{R} \cup\{+\infty\}$, defined in the following way:

Definition 1.4 Let us associate with any exercise time $T$ the tychastic system

$$
\begin{array}{ll}
\text { (i) } & \forall i=0, \ldots, n, \quad x_{i}^{\prime}(t)=x_{i}(t) \rho_{i}(x(t), v(t)) \\
(\text { ii }) & p^{\prime}(t)=u(t) \\
(\text { iii }) & y^{\prime}(t)=\sum_{i=0}^{n} p_{i}(t) x_{i}(t) \rho_{i}(x(t), v(t))+\langle u(t), x(t)\rangle \\
& \text { where } u(t) \in P(T-t, x(t), p(t)) \& v(t) \in Q(T-t, x(t), p(t))
\end{array}
$$

governing the evolution of the prices, the portfolio and the capital and parameterized by controls $u(\cdot)$ and tyches $v(\cdot)$. The problems are:

(a) find the guaranteed valuation subset $\mathcal{V}^{\sharp} \subset \mathbf{R}_{+} \times \mathbf{R}^{n+1} \times \mathbf{R}^{n+1} \times \mathbf{R}_{+}$of (T, $\left.x, p, y\right)$ made of the exercise time $T$, the initial price $x$, the initial portfolio $p$ and the initial capital $y$ such that there exists a dynamical feedback $(t, x, p) \mapsto \widetilde{u}(t, x, p) \in P(t, x, p)$ such that, for all evolutions of tyches $t \in[0, T] \mapsto v(t) \in Q(t, x(t)), p(t)$, for all solutions to differential equation

$$
\begin{cases}(i) & \forall i=0, \ldots, n, x_{i}^{\prime}(t)=x_{i}(t) \rho_{i}(x(t), v(t)) \\ (i i) & p^{\prime}(t)=\widetilde{u}(T-t, x(t), p(t)) \\ (i i i) & y^{\prime}(t)=\sum_{i=0}^{n} p_{i}(t) x_{i}(t) \rho_{i}(x(t), v(t))+\langle\widetilde{u}(T-t, x(t), p(t)), x(t)\rangle \\ & \text { where } v(t) \in Q(T-t, x(t), p(t))\end{cases}
$$


satisfying $x(0)=x, p(0)=p, y(0)=y$, there exists a time $t^{*} \in[0, T]$ such that the chosen condition (1) is satisfied,

(b) associate with any exercise time $T$, initial price $x$ and any portfolio $p$ the smallest capital $V^{\sharp}(T, x, p):$

$$
V^{\sharp}(T, x, p):=\inf _{(T, x, p, y) \in \mathcal{V}^{\sharp}} y
$$

The function $(T, x, p) \mapsto V^{\sharp}(T, x, p)$ is called the guaranteed valuation function of the portfolio, i.e., the minimal initial capital y satisfying the two constraints (1).

(c) Knowing this function, associate the cheapest capital

$$
V_{0}^{\sharp}(T, x):=\inf _{p \in D(T, x)} V^{\sharp}(T, x, p)
$$

in terms of the exercise time and the initial price only.

2. The Regulation Map $\Gamma: \mathbb{R}_{+} \times \mathbb{R}^{n+1} \times \mathbb{R}^{n+1} \leadsto \mathbb{R}^{n+1}$ containing all the dynamical feedbacks

$$
\widetilde{u}(t, x, p) \in \Gamma(t, x, p)
$$

governing the evolutions of portfolios through system 77 (ii): $p^{\prime}(t)=\widetilde{u}(T-t, x(t), p(t))$ satisfying the chosen rule (3) whatever the tyches $v(t) \in Q(T-t, x(t), p(t))$.

\subsection{A Numerical Example}

Even before explaining how the answers to these questions are obtained through the properties of guaranteed capture basins provided by viability theory, we now adapt to the case of transaction costs the Basin Capture Algorithm designed for evaluating options in the case of self-financed portfolio without transaction costs in [62, Pujal \& Saint-Pierre] and [61, Pujal].

For these numerical applications we only consider the case $n=1$ of a single risky asset. We drop the index 1 mentioning the risky asset. Let $x_{K}>0$ be the exercise price and $\bar{p}$ the maximal amount of shares of the risky asset. We take

$$
D(t, x):=\left\{\begin{array}{clll}
{[0, \bar{p}]} & \text { if } & x \in\left[0,2 x_{K}\right] \\
\emptyset & \text { if } & x \notin\left[0,2 x_{K}\right]
\end{array}\right.
$$

The simplest example of tychastic map is obtained when we take $\rho(x, v):=(\rho+v) x$ and $Q(t, x, p):=$ $[-\tau,+\tau]$, where $\tau \geq 0$ describes some measure of versatility (tychastic volatility).

We also assume that $r:=\rho_{0}\left(x_{0}\right)$ is a constant interest rate of the bond and that there are no transaction costs on the bond $\left(\delta_{0}=0\right)$. The evolutions of the price of the bound and of the shares of the bond are independent of the evolutions of the prices and shares of risky assets and of the capital $y$, that are governed by the tychastic control system of 3 equations (instead of 5 equations)

$$
\begin{cases}(i) & x^{\prime}(t)=x(t)(\rho+v(t)) \\ (\text { ii }) & p^{\prime}(t)=u(t) \\ (\text { iii }) & y^{\prime}(t)=y(t) r-p(t) x(t)(r-(\rho+v(t)))-\delta|u(t)| x(t) \\ & \text { where } x(t) \in\left[0,2 x_{K}\right], \quad p(t) \in[0, \bar{p}], \quad|u(t)| \leq \gamma \& v(t) \in[-\tau,+\tau]\end{cases}
$$


We take the classical contingent claim function: $\mathbf{u}\left(x_{0}, x\right):=\max \left(0, x-x_{K}\right)$. We restrict our numerical examples to the European Options (see [61, Pujal] and [62, Pujal \& Saint-Pierre] for other kinds of options in the self-financing case).

We apply the Capture Basin Algorithm in the case of portfolio with transaction costs replicating an European Option in the following cases:

- $T=1, x_{K}=100, \tau:=0.3, r=0, \rho=0.1$.

- $\delta \in\{0.00,0.01,0.02,0.03,0.04,0.05,0.06,0.07,0.08,0.09,0.10\}$, (rate of transaction cost)

The graphs of the approximations of the regulation map $(t, x, p) \mapsto \Gamma(t, x, p)$ are represented in Figure 2 for several values of $t$.

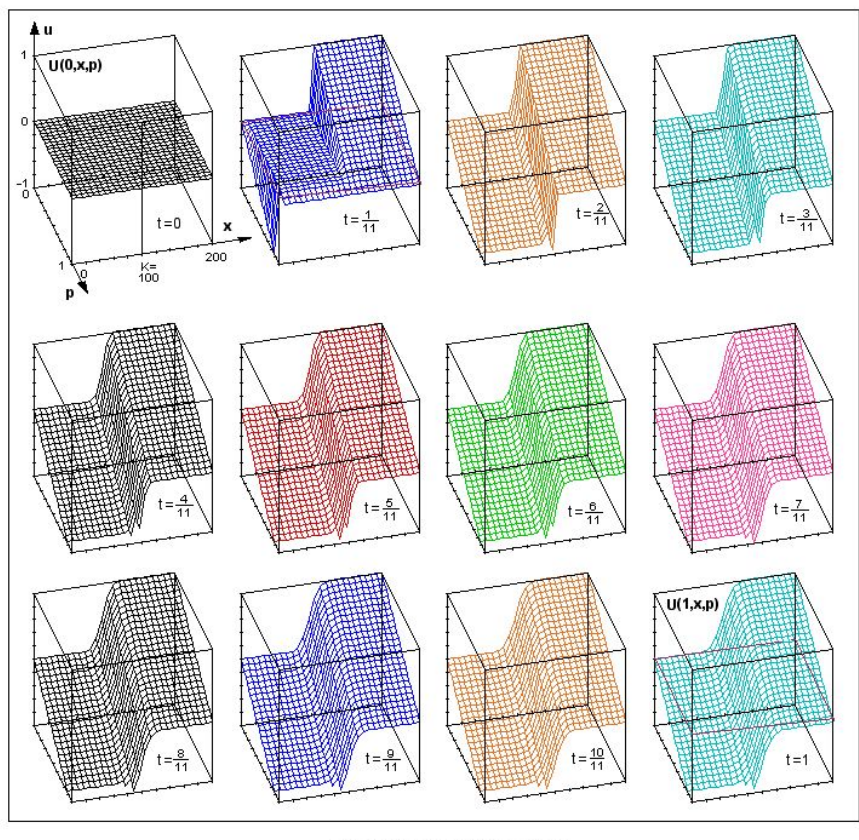

Optimal Buy \& Sell strategy $\mathbf{U}(\mathbf{t}, \mathbf{x}, \mathbf{p})$

$t$ : time left until exercice time

Figure 2: $(t, x, p) \mapsto \Gamma(t, x, p)$ providing the discrete adjustment law $p^{\prime}(t) \in \Gamma(T-t, x(t), p(t))$ providing the variations of the amount of shares of the risky asset in the replicating portfolio. We observe that when $t=T$ is the exercise time, then $p^{\prime}(t)=0$ and no transaction can occur at that time, naturally. We observe that $p^{\prime}(t) \leq 0$ for prices well below the exercise price and that $p^{\prime}(t) \geq 0$ for prices well above the exercise price. 
In Figures 3 and 4 , valuation functions $(x, p) \mapsto V^{\sharp}(T, x, p)$ are computed for different values of $T$. Each graph, corresponding to a given discrete exercise time, provides the value of the option when the price of the risky asset is $x$ and the number of shares of this asset in the portfolio is $p$. They are represented separately in Figure 3 (for case $\delta=1 \%$ ) and superimposed on each other in Figure 4 (with a high value of the transaction $\operatorname{cost} \delta$ for a better view).

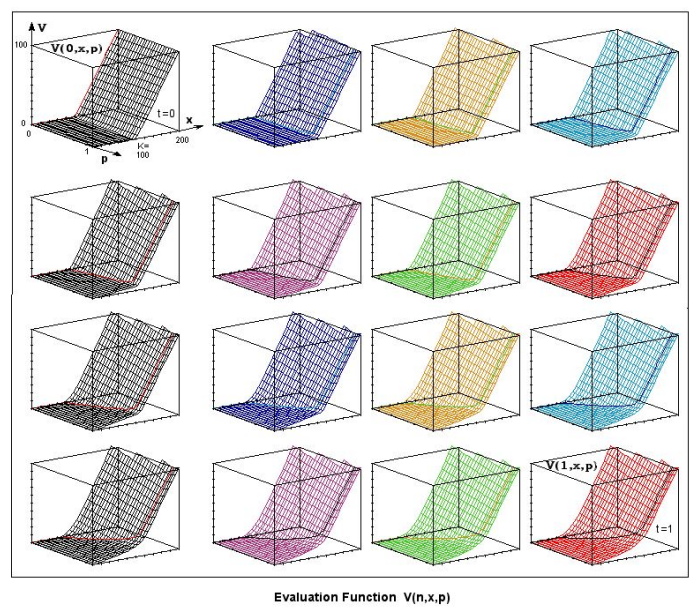

Figure 3: Valuation functions $(x, p) \mapsto V^{\sharp}(T, x, p)$ are computed for different values of $T(\delta=1 \%)$.

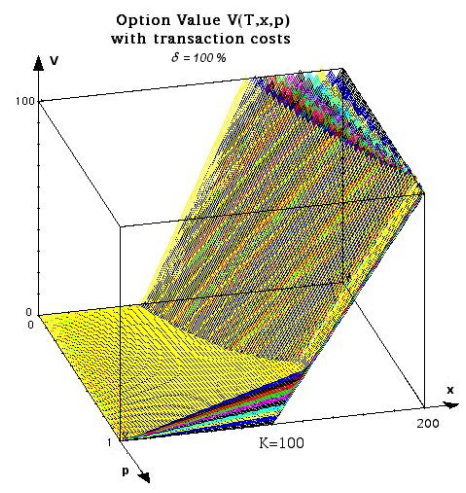

Figure 4: Superimposed valuation functions $(x, p) \mapsto V^{\sharp}(T, x, p)$ are computed for different values of $T$ ((with a high value of the transaction cost $\delta$ for a better view)). 
Figure 5 shows the graph of the valuation function $(x, p) \mapsto V^{\sharp}(T, x, p)$ and the graph of the minimal capital valuation $x \mapsto V_{0}^{\sharp}(T, x):=\inf _{p \in[0, \bar{p}]} V^{\sharp}(T, x, p)$ that is projected in the plane $(x, y)$ in Figure 6

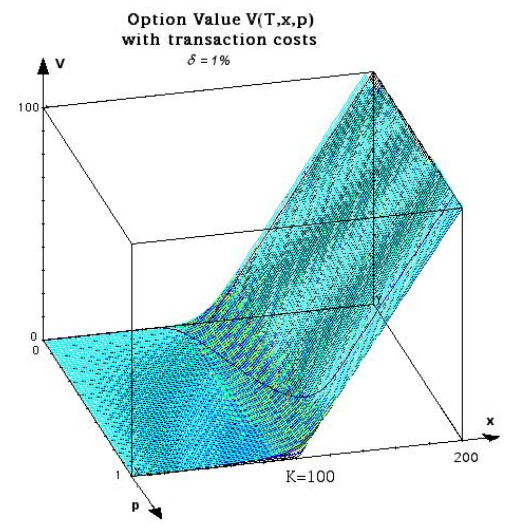

Figure 5: This is the graph of $(x, p) \mapsto V^{\sharp}(T, x, p)$ for $\delta=1 \%$. The graph of the minimal capital valuation function $x \mapsto V_{0}^{\sharp}(T, x):=\inf _{p \in[0, \bar{p}]} V^{\sharp}(T, x, p)$ is represented.

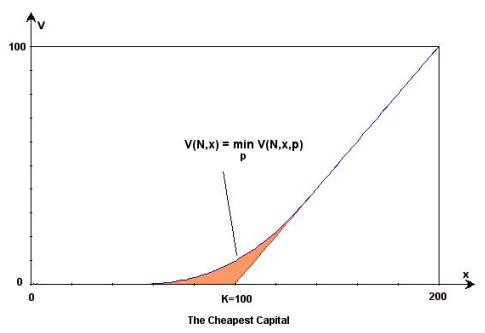

Figure 6: Graph of the minimal capital valuation function $x \mapsto V_{0}^{\sharp}(T, x)$. 


\subsection{Hamilton-Jacobi Inequalities and Regulation Maps}

Establishing partial differential equations à la Black \& Scholes is no longer the starting point of the model, but a property of the valuation function that is derived according the viability/capturability story we told above. This partial differential equation is only useful for defining analytically the dynamics $\Gamma$ of the adjustment law, because we do not need it for computing it by the Capture Basin Algorithm. Since the capture basin and the viability kernel algorithms allow us to compute numerically the valuation function and the evolution of the portfolio, we do not need any longer to solve numerically this type of partial differential equations.

The valuation function $V^{\sharp}$ is actually a solution $\mathbf{v}$ to the nonlinear Hamilton-Jacobi-Isaacs partial differential equation

$$
-\frac{\partial \mathbf{v}(t, x, p)}{\partial t}+\inf _{u \in P(t, x, p)} \sum_{i=0}^{n}\left(\frac{\partial \mathbf{v}(t, x, p)}{\partial p_{i}}-x_{i}\right) u_{i}+\sup _{v \in Q(t, x, p)}\left(\sum_{i=0}^{n}\left(\frac{\partial \mathbf{v}(t, x, p)}{\partial x_{i}}-p_{i}\right) x_{i} \rho_{i}(x, v)\right)=0
$$

satisfying the initial condition

$$
\mathbf{v}(0, x)=\mathbf{u}(x)
$$

on the subset

$$
\begin{gathered}
\Omega_{(\mathbf{b}, \mathbf{c})}(\mathbf{v}):=\left\{(t, x, p) \in \mathbf{R}_{+} \times \mathbf{R}^{n+1} \times \mathbf{R}^{n+1}\right. \text { such that } \\
\\
\mathbf{b}(t, x, p) \leq \mathbf{v}(t, x, p)<\mathbf{c}(t, x, p)\}
\end{gathered}
$$

which depends of the unknown function ${ }^{6} \mathbf{v}$.

For instance, these subsets boil down to

\section{European Case:}

$$
\Omega_{\left(\mathbf{0}, \mathbf{u}_{\infty}\right)}(\mathbf{v}):=\{(t, x, p) \mid t>0 \& \mathbf{v}(t, x, p) \geq 0\}
$$

\section{American Case}

$$
\Omega_{(\mathbf{u}, \mathbf{u} \infty)}(\mathbf{v}):=\{(t, x, p) \mid t>0 \& \mathbf{v}(t, x, p) \geq \mathbf{u}(x)\}
$$

\section{Kairotic Case}

$$
\Omega_{(\mathbf{0}, \mathbf{u})}(\mathbf{v}):=\{(t, x, p) \mid t>0 \& \mathbf{u}(x)>\mathbf{v}(t, x, p) \geq 0\}
$$

Knowing the derivatives of the guaranteed valuation function $V^{\sharp}$, we derive the regulation map $\Gamma$, equal to

$$
\left\{\begin{array}{l}
\Gamma(t, x, p):=\{u \in P(t, x, p) \text { such that } \\
\left.-\frac{\partial V^{\sharp}(t, x, p)}{\partial t}+\sum_{i=0}^{n}\left(\frac{\partial V^{\sharp}(t, x, p)}{\partial p_{i}}-x_{i}\right) u_{i}+\sup _{v \in Q(t, x, p)}\left(\sum_{i=0}^{n}\left(\frac{\partial V^{\sharp}(t, x, p)}{\partial x_{i}}-p_{i}\right) x_{i} \rho_{i}(x, v)\right)=0\right\}
\end{array}\right.
$$

Actually, the solution of the above partial differential equation is taken in the "contingent sense", or, by duality, in the "viscosity sense", as it is explained later.

\footnotetext{
${ }^{6}$ In this case, such problems are called variational inequalities (see for instance [25, Bensoussan \& Lions J.-L.]).
} 
Example Let us consider the simplest case of tychastic uncertainty:

$$
\forall i=1, \ldots, n, \quad \rho_{i}(x, v):=\rho_{i}(x)+v_{i} \text { where } v_{i} \in\left[-\tau_{i},+\tau_{i}\right]
$$

where $\tau_{i}$ denotes the versatility (tychastic volatility) of the $i$ th asset. form

In this case, the above Hamilton-Jacobi-Isaacs partial differential equation can be written in the

$$
\left\{\begin{array}{l}
-\frac{\partial \mathbf{v}(t, x, p)}{\partial t}+\inf _{u \in P(t, x, p)} \sum_{i=0}^{n}\left(\frac{\partial \mathbf{v}(t, x, p)}{\partial p_{i}}-x_{i}\right) u_{i} \\
\sum_{i=0}^{n}\left(\frac{\partial \mathbf{v}(t, x, p)}{\partial x_{i}}-p_{i}\right) x_{i} \rho_{i}(x)+\sum_{i=1}^{n} \tau_{i} x_{i}\left|\frac{\partial \mathbf{v}(t, x, p)}{\partial x_{i}}-p_{i}\right|=0
\end{array}\right.
$$

Remark: Letting the tychastic volatilities grow to $\infty$. When the tychastic volatilities $\tau_{i} \rightarrow+\infty$, we obtain (formally) that

$$
\forall i=1, \ldots, n, \quad p_{i}=\frac{\partial \mathbf{v}(t, x, p)}{\partial x_{i}}
$$

a formula familiar in in the finance literature under the name of "Greeks". Writing

$$
p_{0} x_{0}=\mathbf{v}(t, x, p)-\sum_{i=1}^{n} p_{i} x_{i}=\mathbf{v}(t, x, p)-\sum_{i=1}^{n} \frac{\partial \mathbf{v}(t, x, p)}{\partial x_{i}} x_{i}
$$

the above partial differential equation boils down to

$$
\left\{\begin{array}{c}
-\frac{\partial \mathbf{v}(t, x, p)}{\partial t}+\inf _{u \in P(t, x, p)} \sum_{i=0}^{n}\left(\frac{\partial \mathbf{v}(t, x, p)}{\partial p_{i}}-x_{i}\right) u_{i} \\
+\rho_{0}\left(x_{0}\right) \sum_{i=0}^{n} \frac{\partial \mathbf{v}(t, x, p)}{\partial x_{i}} x_{i}-\rho_{0}\left(x_{0}\right) \mathbf{v}(t, x, p)=0
\end{array}\right.
$$

This is (in the case with transaction costs) the first-order partial differential equation derived by Pierre Bernhard (under the name of the "naive theory") in [28, 29, Bernhard] in the "Black \& Scholes style". It is obtained when the uncertainties are maximal $\left(\tau_{i}=+\infty\right)$, through a limiting procedure of the case when the tychastic uncertainty is limited in the sense that the tyche are bounded.

\subsection{Analytical Formulas for the Valuation Functions}

We shall first provide a formula for the valuation function that states that it is the valuation function of a two person dynamical game:

We associate with the contingent claim function $\mathbf{u}$ the functional

$$
\left\{\begin{array}{l}
J_{\mathbf{u}}(t ;(x(\cdot), p(\cdot), v(\cdot)) ; \widetilde{u})(T, x, p):=e^{-\int_{0}^{t} \rho_{0}\left(x_{0}(s)\right) d s} \mathbf{u}(x(t), p(t)) \\
+\int_{0}^{t} e^{-\int_{0}^{\tau} \rho_{0}\left(x_{0}(s)\right) d s}\left(\sum_{i=0}^{n} p_{i}(\tau) x_{i}(\tau)\left(\rho_{0}\left(x_{0}(\tau)\right)-\rho_{i}\left(x_{i}(\tau), v(\tau)\right)\right)-\langle\widetilde{u}(T-\tau, x(\tau)), x(\tau)\rangle\right) d \tau
\end{array}\right.
$$


We set

$$
\left\{\begin{array}{l}
I_{\mathbf{0}}(t ;(x(\cdot), p(\cdot), v(\cdot)) ; \widetilde{u})(t, x, p):= \\
\sup _{r \in[0, t]} \int_{0}^{r} e^{-\int_{0}^{r} \rho_{0}\left(x_{0}(s)\right) d s}\left(\sum_{i=0}^{n} p_{i}(\tau) x_{i}(\tau)\left(\rho_{0}\left(x_{0}(\tau)\right)-\rho_{i}\left(x_{i}(\tau), v(\tau)\right)\right)-\langle\widetilde{u}(T-\tau, x(\tau)), x(\tau)\rangle\right) d \tau
\end{array}\right.
$$

We shall associate with it and with each of the three rules of the game the three corresponding valuation function 7 .

1. European Options: We obtain

$$
\left\{\begin{array}{l}
V_{\left(\mathbf{0}, \mathbf{u}_{\infty}\right)}^{\sharp}(T, x, p)=\inf _{\widetilde{u}(\cdot) \in P(t, x, p)} \sup _{(x(\cdot), p(\cdot), v(\cdot)) \in \mathcal{C}_{\tilde{u}}(x, p)} \\
\max \left[J_{\mathbf{u}}(T ;(x(\cdot), p(\cdot), v(\cdot)) ; \widetilde{u})(T, x, p), I_{\mathbf{0}}(T ;(x(\cdot), p(\cdot), v(\cdot)) ; \widetilde{u})(T, x, p)\right]
\end{array}\right.
$$

2. American Options: We obtain

$$
\left\{\begin{array}{l}
V_{\left(\mathbf{u}^{\prime} \mathbf{u}_{\infty}\right)}^{\sharp}(T, x, p)=\inf _{\widetilde{u}(\cdot) \in P(t, x, p)} \sup _{(x(\cdot), p(\cdot), v(\cdot)) \in \mathcal{C}_{\tilde{u}}(x, p)} \\
\sup _{t \in[0, T]} \max \left[J_{\mathbf{u}}(t ;(x(\cdot), p(\cdot), v(\cdot)) ; \widetilde{u})(T, x, p), I_{0}(t ;(x(\cdot), p(\cdot), v(\cdot)) ; \widetilde{u})(T, x, p)\right]
\end{array}\right.
$$

3. Kairotic options: We obtain

$$
\left\{\begin{array}{l}
V_{(\mathbf{0}, \mathbf{u})}^{\sharp}(T, x, p)=\inf _{\widetilde{u}(\cdot) \in P(t, x, p)} \sup _{(x(\cdot), p(\cdot), v(\cdot)) \in \mathcal{C}_{\tilde{u}}(x, p)} \\
\inf _{t \in[0, T]} \max \left[J_{\mathbf{u}}(t ;(x(\cdot), p(\cdot), v(\cdot)) ; \widetilde{u})(T, x, p), I_{\mathbf{0}}(t ;(x(\cdot), p(\cdot), v(\cdot)) ; \widetilde{u})(T, x, p)\right]
\end{array}\right.
$$

\section{The Viability/Capturability Strategy}

We shall prove that the epigraph of the guaranteed valuation function is the guaranteed viable-capture basin of a target viable in a constrained set under an auxiliary dynamical game that we shall construct. Both the target and the constrained sets are epigraphs of functions associated with the contingent claim function $\mathbf{u}$ and one of the three rules (European, American, Kairotic) we have singled-out, as we shall see later. Once this task is completed, we can

1. gather the properties of guaranteed capture basins of targets under tychastic control systems at this general level, and in particular, study the tangential conditions enjoyed by the guaranteed viable-capture basins (see Theorem 2.6 below),

2. adapt the Capture Basin Algorithm for computing numerically the (epigraph of) the guaranteed valuation function and the (graph of) the regulation map, by discretizing the problem and by using algorithms devised in [66, Saint-Pierre], 62, Pujal \& Saint-Pierre] and [61, Pujal].

3. and use set-valued analysis and nonsmooth analysis for translating the general results of guaranteed viable-capture basins to the corresponding results on our option dynamic valuation and management problem, in particular translating tangential conditions to give a meaning to the concept of a generalized solution (Frankowska's episolutions or, by duality, viscosity solutions) to Hamilton-Jacobi-Isaacs variational inequalities (see Theorems 2.7 and 2.8 below).

\footnotetext{
${ }^{7}$ The notations $\left(\mathbf{0}, \mathbf{u}_{\infty}\right),\left(\mathbf{u}, \mathbf{u}_{\infty}\right),(\mathbf{0}, \mathbf{u})$ labelling the European, America and Kairotic option rules respectively will be explained and justified later.
} 
The approach we propose distinguishes clearly the rules of the games, such as the rules (1), which appear in the target and the constrained set of the auxiliary problem, from the nature of the dynamics governing the evolution of the prices. The choice of these dynamics predicting or extrapolating the dynamics of the prices is another problem that we do not address here.

However, our hope is to adapt the viability/capturability strategy we adopt here to other kinds of dynamics whenever it has not be done yet:

1. discrete stochastic evolutions. This viability/capturability approach has already be used by J. Zabczyk for European and American options in the framework of discrete stochastic models under the name of controllability and strong controllability ([75, 76, Zabczyk])

2. continuous stochastic evolutions. The capturability approach has already been used by Soner \& Touzi for European options in the framework of stochastic control problems under the name of stochastic targets ([69, 70, 71, Soner \& Touzi]). However, option pricing with transaction costs to quote [68, Soner, Shreve \& Cvitanic] presents difficulties in the stochastic case.

Viability and Invariance Theorems have been adapted to the stochastic case, as in [11, 12, Aubin \& Da Prato], [13, Aubin, Da Prato \& Frankowska], 15, Aubin \& Doss], 41, 42, 43, Da Prato \& Frankowska], [23, 24, Bardi \& Goatin], [35, Buckdahn, Cardaliaguet \& Quincampoix], [32, 33. Buckdahn, Quincampoix \& Rascanu], [34, Buckdahn, Peng, Quincampoix \& Rainer], [50, Gautier \& Thibault], [56, Kisielewicz], etc.

3. history dependent (path dependent) evolutions They involve the case when the evolution of financial asset prices is governed by an history dependent (path dependent) dynamical system as a prediction mechanism. For instance, 20, Aubin \& Haddad] studies the dynamical valuation and management of a portfolio (replicating for instance European, American and other options) depending upon such a prediction mechanism (instead of an uncertain evolution of prices, stochastic or tychastic), using in particular results of [51, 52, 53, Haddad], partly presented in Chapter 12 of [3, Aubin]. The valuation functionals are solutions of kinds of Hamilton-Jacobi equations involving "Clio derivatives" of functionals (on the history of an evolution),

4. impulse tychastic evolutions, allowing to take into account payments of dividends using results of [5, 6, 17, Aubin], [18, 19, Aubin \& Haddad], 21, Aubin, Lygeros, Quincampoix, Sastry \& Seube] in impulse control and hybrid systems (see for instance [26, Bensoussan \& Menaldi], 67, Saint-Pierre], and, in the stochastic case, [73, Zabczyk], among many other references).

In this paper, we shall treat only the tychastic control approach and answer two types of questions:

1. provide a formula of the valuation function and uncover the underlying criterion to be optimized in a tychastic way.

2. prove that the valuation function is the solution of Hamilton-Jacobi-Isaacs variational inequalities and derive the adjustment law.

For that purpose, we recall the minimal number of concepts of viability theory needed to prove the results mentioned above to derive in the final section the results presented in the first section. 


\subsection{Absorption Basins under Tychastic systems}

We denote by $X$ and $\mathcal{V}$ two finite dimensional vector spaces, and we introduce a single-valued map $f: X \times \mathcal{V} \leadsto X$ and a tychastic set-valued map $Q: X \leadsto \mathcal{V}$. A tychastic system is governed by a singlevalued map $f$ generating a differential equation parameterized by tyches ranging over a tychastic map $Q$ :

$$
\begin{cases}(i) & x^{\prime}(t)=f(x(t), v(t)) \\ (\text { ii }) & v(t) \in Q(x(t))\end{cases}
$$

We denote by $\mathcal{S}(x)$ the subset of evolutions starting at $x$ governed by the tychastic system.

Definition 2.1 Let $K \subset X$ be a environment and $C \subset K$ be a target.

1. The subset $\operatorname{Inv}_{\mathcal{S}}(K, C)$ of initial states $x_{0} \in K$ such that all evolutions $x(\cdot) \in \mathcal{S}\left(x_{0}\right)$ starting at $x_{0}$ are viable in $K$ for all $t \geq 0$ or viable in $K$ until they reach $C$ in finite time is called the invariance kernel of $K$ with target $C$ under $\mathcal{S}$.

When the target $C=\emptyset$ is the empty set, we say that $\operatorname{Inv}_{\mathcal{S}}(K):=\operatorname{Inv}_{\mathcal{S}}(K, \emptyset)$ is the invariance kernel of $K$.

2. The subset $\operatorname{Abs}_{\mathcal{S}}(K, C)$ of initial states $x_{0} \in K$ such that all evolutions $x(\cdot) \in \mathcal{S}\left(x_{0}\right)$ starting at $x_{0}$ are viable in $K$ until they reach $C$ in finite time is called the absorption basin of $K$ with target $C$ under $\mathcal{S}$.

When $K=X$ is the whole space, we say that $\operatorname{Abs}_{\mathcal{S}}(X, C)$ is the absorption basin of $C$.

We say that

1. a subset $K$ is invariant under $\mathcal{S}$ if $K=\operatorname{Inv}(K)$,

2. $K$ is invariant outside a target $C \subset K$ under the evolutionary system $\mathcal{S}$ if $K=\operatorname{Inv}(K, C)$ and that $K$ is invariant under $\mathcal{S}$ if $K=\operatorname{Inv}(K)$,

3. $C$ is separated in $K$ if $C=\operatorname{Inv}(K, C)$.

It easy to check that whenever the complement $K \backslash C:=K \cap \complement C$ of the target $C$ in the environment is a REPELLER (this means that all evolutions starting from $K \backslash C$ leave it in finite time), the invariance kernel with target $C$ and the absorption basin of target $C$ coincide. This will be the case of the auxiliary systems introduced later for deriving the announced results.

We deduce from [9, Aubin \& Catté] the following characterization of invariance kernels:

Theorem 2.2 The invariance kernel $\operatorname{Inv}_{\mathcal{S}}(K, C)$ of $K$ outside the target $C$ is the unique subset between $C$ and $K$ that is both

1. invariant outside $C$ (and is the largest subset $D \subset K$ viable outside $C$ ),

2. separated in $K$ (and is the smallest subset $D \supset C$ isolated in $K$ ): 


$$
\operatorname{Inv}_{\mathcal{S}}\left(K, \operatorname{Inv}_{\mathcal{S}}(K, C)\right)=\operatorname{Inv}_{\mathcal{S}}(K, C)=\operatorname{Inv}_{\mathcal{S}}\left(\operatorname{Inv}_{\mathcal{S}}(K, C), C\right)
$$

and thus, the unique bilateral fixed point $D$ between $C$ and $K$ in the sense that

$$
D=\operatorname{Inv}_{\mathcal{S}}(K, D)=\operatorname{Inv}_{\mathcal{S}}(D, C)
$$

The same properties are shared by the absorption basins.

The Invariance Theorem characterizes absorption basins through tangential conditions involving tangent cones (contingent cone) to a subset $K$ at a point $x \in K$, introduced in the early 1930's independently by Bouligand and Severi : They adapt to any subset the concept of tangent space to manifolds: A direction $v \in X$ belongs to $T_{K}(x)$ if there exist sequences $h_{n}>0$ and $v_{n} \in X$ converging to 0 and $v$ respectively such that

$$
\forall n \geq 0, \quad x+h_{n} v_{n} \in K
$$

Theorem 2.3 Assume that the dynamics $f$ and the tychastic map $Q$ are Lipschitz and that the target $C$ and the environment $K$ are closed. Then the absorption basin $\operatorname{Abs}_{\mathcal{S}}(K, C)$ is the largest closed subset $D$ between $C$ and $K$ satisfying the tangential condition

$$
\forall x \in K \backslash D, \quad \forall v \in Q(x), \quad f(x, v) \in T_{D}(x)
$$

Furthermore, the Viability Theorem implies that if the assumptions of Theorem 2.3 are satisfied, if the tychastic system is Marchaud and that if the interior of the target is closed, that the absorption basin $\operatorname{Abs}_{\mathcal{S}}(K, C)$ is the unique subset $D$ between $C$ and $K$ satisfying tangential condition (15) and tangential condition

$$
\forall x \in \operatorname{Int}(K) \backslash \operatorname{Int}(D), \quad \exists v \in Q(x) \text { such that } f(x, v) \in T_{\overline{\mathrm{C} D}}(x)
$$




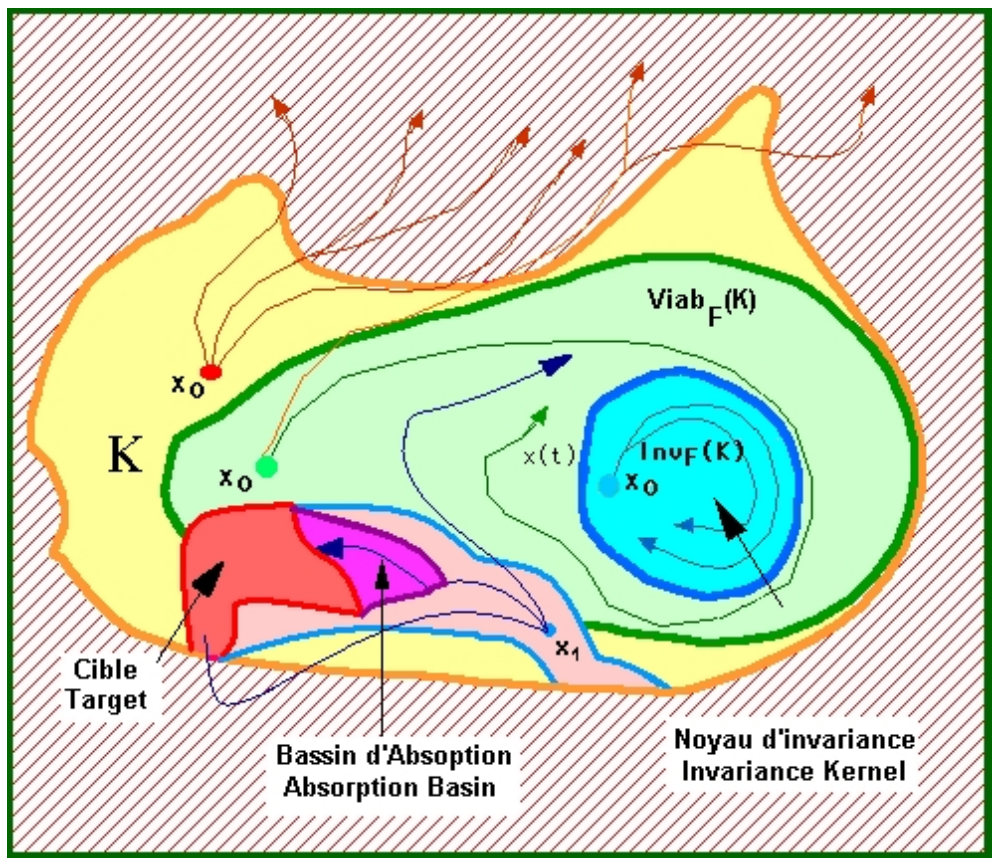




\subsection{Guaranteed Viable-Capture Basins}

We summarize the main results on guaranteed viability/capturability of a target under dynamical games that we need to prove the results announced in the preceding section.

We denote by $X, \mathcal{U}$ and $\mathcal{V}$ three finite dimensional vector spaces, and we introduce a single-valued map $f: X \times \mathcal{U} \times \mathcal{V} \leadsto X$, a cybernetic set-valued map $P: X \leadsto \mathcal{U}$ and a tychastic set-valued map $Q: X \leadsto \mathcal{V}$.

We consider a dynamical game described by

$$
\begin{cases}(i) & x^{\prime}(t)=f(x(t), u(t), v(t)) \\ (\text { ii }) & u(t) \in P(x(t)) \\ (\text { iii }) & v(t) \in Q(x(t))\end{cases}
$$

which is, so to speak, a control system regulated by two parameters, $u(t)$ and $v(t)$, the first one regarded as a regulating parameter, controlled by a player, the second one regarded as a perturbation, or a disturbance, or a tyche, chosen in a unknown way by "Nature".

We introduce a class $\widetilde{\mathcal{P}}$ of continuous selections $x \mapsto \widetilde{u}(x) \in P(x)$, that are used as feedbacks or strategies by the player controlling the parameters $u$.

We associate with such a feedback $\widetilde{u}(x) \in P(x)$ the set $\mathcal{C}_{\widetilde{u}}(x)$ of solutions $(x(\cdot), v(\cdot)) \in \mathcal{C}(0, \infty ; X) \times$ $L^{1}(0, \infty ; \mathcal{U})$ to the parameterized system

$$
\begin{cases}(i) & x^{\prime}(t)=f(x(t), \widetilde{u}(x(t)), v(t)) \\ (\text { ii }) & v(t) \in Q(x(t))\end{cases}
$$

starting at $x$.

We may identify the above dynamical game with the set-valued map $(x, \widetilde{u}) \leadsto \mathcal{C}_{\widetilde{u}}(x)$, that we regard as an evolutionary game.

Definition 2.4 Let $C \subset K \subset X$ be two subsets, $C$ being regarded as a target, $K$ as a constrained set.

We denote by $\operatorname{Abs}_{\tilde{u}}(K, C)$ the invariance-absorption basin of $C$ in $K$, subset of initial states $x_{0} \in K$ such that $C$ is reached in finite time before possibly leaving $K$ by all solutions to (18) starting at $x_{0}$.

The subset

$$
\left[\operatorname{Capt}_{P} \operatorname{Abs}_{Q}\right](K, C):=\bigcup_{\widetilde{u} \in \widetilde{\mathcal{P}}} \operatorname{Abs}_{\widetilde{u}}(K, C)
$$

of elements $x \in K$ such that there exists a feedback $\widetilde{u} \in \widetilde{\mathcal{P}}$ such that for every solutions $(x(\cdot), v(\cdot)) \in$ $\mathcal{C}_{\widetilde{u}}(x)$, there exists $t^{*} \in \mathbf{R}_{+}$satisfying the viability/capturability conditions

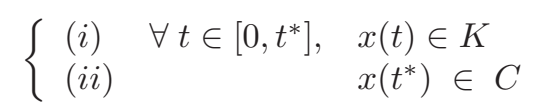

is called the guaranteed viable-capture basin of a target under the evolutionary game $(x, \widetilde{u}) \leadsto \mathcal{C}_{\widetilde{u}}(x)$ defined on $X \times \widetilde{\mathcal{P}}$ (that, naturally, depends upon the choice of the family $\widetilde{\mathcal{P}}$ of feedbacks).

We deduce from [9, Aubin \& Catté] that 
Theorem 2.5 The guaranteed viable-capture basin $\left[\mathrm{Capt}_{P} \mathrm{Abs}_{Q}\right](K, C)$ is the largest subset between $C$ and $K$ is the largest fixed point of the map $D \mapsto\left[\operatorname{Capt}_{P} \operatorname{Abs}_{Q}\right](D, C)$.

Consequently, the guaranteed viable-capture basin satisfies

$$
\left[\operatorname{Capt}_{P} \operatorname{Abs}_{Q}\right](K, C)=\left[\operatorname{Capt}_{P} \operatorname{Abs}_{Q}\right]\left(\left[\operatorname{Capt}_{P} \operatorname{Abs}_{Q}\right](K, C), C\right)
$$

In other words, it is the largest subset of elements $x \in K$ such that there exists a feedback $\widetilde{u} \in \widetilde{\mathcal{P}}$ such that for every solutions $(x(\cdot), v(\cdot)) \in \mathcal{C}_{\widetilde{u}}(x)$, there exists $t^{*} \in \mathbf{R}_{+}$satisfying the viability/capturability conditions.

We shall assume that the dynamical game (17) is Lipschitz in the sense that the set-valued maps $P$ and $Q$ are Lipschitz with compact values and that the single -valued map $f$ is Lipschitz with closed values.

Let $\widetilde{\mathcal{P}}_{\lambda}$ be the set of Lipschitz selections with constant $\lambda$ of the set-valued map $P$ : for every $x$, $\widetilde{u}(x) \in P(x)$.

The subset

$$
\left[\operatorname{Capt}_{P_{\lambda}} \operatorname{Abs}_{Q}\right](K, C):=\bigcup_{\widetilde{u} \in \widetilde{\mathcal{P}}_{\lambda}} \operatorname{Abs}_{\widetilde{u}}(K, C)
$$

is called the $\lambda$-guaranteed viable-capture basin of a target under the evolutionary game $(x, \widetilde{u}) \leadsto \mathcal{C}_{\widetilde{u}}(x)$ associated with the dynamical game (17).

One can prove that when the game is Lipschitz, the set-valued map $(x, \widetilde{u}) \in X \times \widetilde{\mathcal{P}}_{\lambda} \leadsto \mathcal{C}_{\widetilde{u}}(x) \subset$ $\mathcal{C}(0, \infty ; X)$ is lower semicontinuous and consequently, that the $\lambda$-guaranteed viable-capture basin is closed.

Using the Viability and the Invariance Theorems, one can prove the following tangential properties of guaranteed viability kernels with targets:

Theorem 2.6 Let us assume that the dynamical game $(P, Q, f)$ is Lipschitz, that $C \subset K$ and $K$ are closed subsets of $X$ and that $K \backslash C$ is a repeller under all the maps $(x, \widetilde{u}) \leadsto \mathcal{C}_{\widetilde{u}}(x)$.

Then the $\lambda$-guaranteed viable-capture basin $\left[\operatorname{Capt}_{P_{\lambda}} \mathrm{Abs}_{Q}\right](K, C)$ of target $C$ viable in $K$ is the largest of the closed subsets $D$ satisfying $C \subset D \subset K$ and

1. the tangential property

$$
\forall x \in D \backslash C, \exists u \in P(x) \text { such that } \forall v \in Q(x), \quad f(x, u, v) \in T_{D}(x)
$$

2. there exists a $\lambda$-Lipschitz selection of the guaranteed regulation map $\Gamma_{D}$ defined by

$$
\forall x \in D \backslash C, \Gamma_{D}(x):=\left\{u \in P(x) \mid f(x, u, Q(x)) \subset T_{D}(x)\right\}
$$

This theorem is a restatement of Theorems 9.2.14 and 9.2.18 of [4, Aubin, Chapter 9].

\footnotetext{
${ }^{8}$ or, the equivalent dual formulation,

$$
\forall x \in D \backslash C, \forall p \in N_{D}(x), \inf _{u \in P(x)} \sup _{v \in Q(x)}\langle f(x, u, v), p\rangle \leq 0
$$

where the (regular) normal cone $N_{D}(x):=T_{D}(x)^{-}$is the polar cone to the contingent cone $T_{D}(x)$.
} 


\subsection{Intertemporal Games}

We introduce the following features:

1. a "Lagrangian"

$$
\mathbf{l}:(x, u, v) \in X \times \mathcal{U} \times \mathcal{V} \mapsto \mathbf{l}(x, u, v) \in \mathbf{R}_{+}
$$

2. two nonnegative extended cost functions $\mathbf{b}$ and $\mathbf{c}$ from $\mathbf{R}_{+} \times X$ to $\mathbf{R}_{+} \cup\{+\infty\}$ satisfying

$$
\forall(t, x) \in \mathbf{R}_{+} \times X, \quad 0 \leq \mathbf{b}(t, x) \leq \mathbf{c}(t, x) \leq+\infty
$$

that we shall extend to cost functions (again denoted by) $\mathbf{b}$ (constrained function) and $\mathbf{c}$ (objective function) from $\mathbf{R} \times X$ to $\mathbf{R}_{+} \cup\{+\infty\}$ by setting

$$
\mathbf{b}(t, x)=\mathbf{c}(t, x):=+\infty \text { whenever } t<0
$$

We next fix an horizon or an exercise time $T$ and associate with it the cost functionals

$$
\left\{\begin{array}{l}
J_{\mathbf{c}}(t ;(x(\cdot), v(\cdot)) ; \widetilde{u})(T, x):=\mathbf{c}(T-t, x(t)) \\
+\int_{0}^{t} \mathbf{l}(x(\tau), \widetilde{u}(x(\tau)), v(\tau)) d \tau
\end{array}\right.
$$

(where $t$ ranges over $[0, T]$ ),

$$
I_{\mathbf{b}}(t ;(x(\cdot), v(\cdot)) ; \widetilde{u})(T, x):=\sup _{s \in[0, t]} J_{\mathbf{b}}(s ;(x(\cdot), v(\cdot)) ; \widetilde{u})(T, x)
$$

and

$$
L_{(\mathbf{b}, \mathbf{c})}(t ;(x(\cdot), v(\cdot)) ; \widetilde{u})(T, x):=\max \left(J_{\mathbf{c}}(t ;(x(\cdot), v(\cdot)) ; \widetilde{u})(T, x), I_{\mathbf{b}}(t ;(x(\cdot), v(\cdot)) ; \widetilde{u})(T, x)\right)
$$

We associate with it the guaranteed valuation function

$$
V_{(\mathbf{b}, \mathbf{c})}^{\sharp}(T, x):=\inf _{\widetilde{u} \in \widetilde{\mathcal{P}}} \sup _{(x(\cdot), v(\cdot)) \in \mathcal{C}_{\tilde{u}}(x)} \inf _{t \in[0, T]} L_{(\mathbf{b}, \mathbf{c})}(t ;(x(\cdot), v(\cdot)) ; \widetilde{u})(T, x)
$$

The function $V_{(\mathbf{b}, \mathbf{c})}^{\sharp}$ is called the guaranteed valuation function associated with $\mathbf{l}$ and the cost functions $\mathbf{b}$ and $\mathbf{c}$.

Let us consider the extended dynamical game of the form:

$$
\begin{cases}(i) & \tau^{\prime}(t)=-1 \\ (i i) & x^{\prime}(t)=f(x(t), u(t), v(t)) \\ (\text { iii }) & y^{\prime}(t)=-\mathbf{l}(x(t), u(t), v(t)) \\ & \text { where } u(t) \in P(x(t)) \& v(t) \in Q(x(t))\end{cases}
$$

We associate with such a feedback $\widetilde{u}(x) \in P(x)$ the set $\mathcal{B}_{\widetilde{u}}(T, x, y)$ of solutions $(T-\cdot, x(\cdot), v(\cdot), y(\cdot))$ to the auxiliary system

$$
\begin{cases}(i) & \tau^{\prime}(t)=-1 \\ (\text { ii }) & x^{\prime}(t)=f(x(t), \widetilde{u}(x(t)), v(t)) \\ (\text { iii }) & y^{\prime}(t)=-\mathbf{l}(x(t), \widetilde{u}(x(t)), v(t)) \\ & \text { where } v(t) \in Q(x(t))\end{cases}
$$


Theorem 2.7 Let us assume that the extended functions $\mathbf{b}$ and $\mathbf{c}$ are nontrivial and non negative.

The guaranteed valuation function $V_{(\mathbf{b}, \mathbf{c})}^{\sharp}$ defined by 20 is related to the guaranteed viable-capture basin $\left[\operatorname{Capt}_{P} \operatorname{Abs}_{Q}\right](\mathcal{E} p(\mathbf{b}), \mathcal{E} p(\mathbf{c})$ of the epigraph $\mathcal{E} p(\mathbf{c})$ of $\mathbf{c}$ under the dynamical game 21) viable in the epigraph $\mathcal{E} p(\mathbf{b})$ of $\mathbf{b}$ :

$$
V_{(\mathbf{b}, \mathbf{c})}^{\sharp}(T, x):=\inf _{(T, x, y) \in\left[\operatorname{Capt}_{P} \operatorname{Abs}_{Q}\right](\mathcal{E} p(\mathbf{b}), \mathcal{E} p(\mathbf{c})} y
$$

Since the guaranteed viable-capture basin

$$
\left[\operatorname{Capt}_{P} \operatorname{Abs}_{Q}\right]\left(\mathcal{E} p(\mathbf{b}), \mathcal{E} p(\mathbf{c}):=\bigcup_{\widetilde{u} \in \widetilde{\mathcal{P}}} \operatorname{Abs}_{\widetilde{u}}(\mathcal{E} p(\mathbf{b}), \mathcal{E} p(\mathbf{c}))\right.
$$

is an union of capture basins, we deduce that

$$
V_{(\mathbf{b}, \mathbf{c})}^{\sharp}(T, x):=\inf _{(T, x, y) \in\left[\operatorname{Capt}_{P} \operatorname{Abs}_{Q}\right](\mathcal{E} p(\mathbf{b}), \mathcal{E} p(\mathbf{c})} y
$$

is the pointwise infimum

$$
V_{(\mathbf{b}, \mathbf{c})}^{\sharp}(T, x)=\inf _{\widetilde{u} \in \widetilde{\mathcal{P}}} U_{(\mathbf{b}, \mathbf{c} ; \widetilde{u})}(T, x)
$$

of the functions

$$
U_{(\mathbf{b}, \mathbf{c} ; \tilde{u})}(T, x):=\inf _{(x, T, y) \in \operatorname{Abs}_{\tilde{u}}(\mathcal{E} p(\mathbf{b}), \mathcal{E} p(\mathbf{c})} y
$$

It remains to use formula

$$
U_{(\mathbf{b}, \mathbf{c} ; \tilde{u})}(T, x)=\sup _{(x(\cdot), v(\cdot)) \in \mathcal{C}_{\tilde{u}}(x)} \inf _{t \in[0, T]} L_{(\mathbf{b}, \mathbf{c})}(t ;(x(\cdot), v(\cdot)) ; \widetilde{u})(T, x)
$$

proved in [61, Pujal] to derive Theorem 2.7.

\subsection{Hamilton-Jacobi-Isaacs Variational Inequalities}

Let us recall that the contingent epiderivative $D_{\uparrow} \mathbf{u}(t, x)$ of $\mathbf{u}$ at $(t, x)$ is defined by

$$
D_{\uparrow} \mathbf{u}(t, x)(\lambda, v):=\liminf _{h \rightarrow 0+, u \rightarrow v} \frac{\mathbf{u}(t+h \lambda, x+h u)-\mathbf{u}(t, x)}{h}
$$

and that

$$
\mathcal{E} p\left(D_{\uparrow} \mathbf{u}(t, x)\right)=T_{\mathcal{E} p(\mathbf{u})}(t, x, \mathbf{u}(t, x))
$$

Since the $\lambda$-guaranteed viable-capture basin is closed under Lipschitz equations, then it is the epigraph of the lower semicontinuous the $\lambda$-guaranteed valuation function

$$
V_{\left(\mathcal{E} p(\mathbf{b}), \mathcal{E} p(\mathbf{c})_{\lambda}\right)}^{\sharp}(T, x):=\inf _{\widetilde{u} \in \widetilde{\mathcal{P}}_{\lambda}} \sup _{(x(\cdot), v(\cdot)) \in \mathcal{C}_{\tilde{u}}(x)} \inf _{t \in[0, T]} L_{(\mathbf{b}, \mathbf{c})}(t ;(x(\cdot), v(\cdot)) ; \widetilde{u})(T, x)
$$


Theorem 2.8 Let us assume that the dynamical game 21) is Lipschitz and that the cost functions $\mathbf{b}$ and $\mathbf{c}$ from $\mathbf{R}_{+} \times X$ to $\mathbf{R}_{+} \cup\{+\infty\}$ are nontrivial, nonnegative and lower semicontinuous. Then the $\lambda$-guaranteed valuation function $V_{(\mathbf{b}, \mathbf{c})_{\lambda}}^{\sharp}$ under the dynamical game 17 is the smallest of the lower semicontinuous solutions $\mathbf{v}$ to the Hamilton-Jacobi-Isaacs contingent inequalities

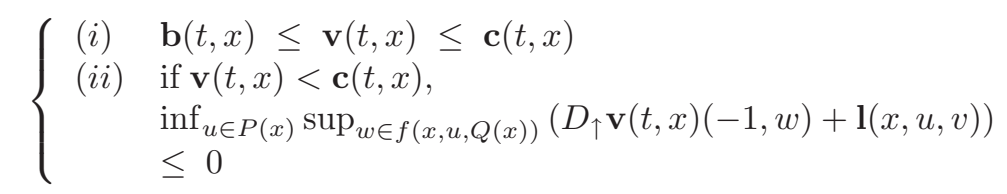

such that there exists a $\lambda$-Lipschitz selection $\widetilde{u}$ of the guaranteed regulation map $\Gamma$ defined by

$$
\left\{\begin{array}{l}
\Gamma(t, x) \\
:=\left\{u \in P(x) \mid \sup _{w \in f(x, u, Q(x))}\left(D_{\uparrow} \mathbf{v}(t, x)(-1, w)+\mathbf{l}(x, u, v)\right) \leq 0\right\}
\end{array}\right.
$$

Proof - It is a consequence of Theorem 2.6 when $K:=\mathcal{E} p(\mathbf{b}), C:=\mathcal{E} p(\mathbf{c})$ and when the dynamical game is the extended dynamical game 21 .

Theorem 2.6 states that the $\lambda$-guaranteed viable-capture basin

$$
\left[\operatorname{Capt}_{P_{\lambda}} \operatorname{Abs}_{Q}\right]_{(\mathcal{E} p(\mathbf{b}), \mathcal{E} p(\mathbf{c}))}
$$

under 21) of the epigraph $\mathcal{E} p(\mathbf{c})$ of $\mathbf{c}$ viable in the epigraph $\mathcal{E} p(\mathbf{b})$ of $\mathbf{b}$ is the largest of the closed subsets $\mathcal{U}$ satisfying $\mathcal{E} p(\mathbf{c}) \subset \mathcal{U} \subset \mathcal{E} p(\mathbf{b})$, the tangential conditions

$$
\left\{\begin{array}{l}
\forall(t, x, y) \in \mathcal{U} \backslash \mathcal{E} p(\mathbf{c}), \exists u \in P(x) \text { such that } \forall w \in f(x, u, Q(x)), \\
(-1, w,-\mathbf{l}(x, u, v)) \in T_{\mathcal{U}}(t, x, y)
\end{array}\right.
$$

and such that there exists a $\lambda$-Lipschitz selection of the guaranteed regulation map $\Gamma_{\mathcal{U}}$ defined by

$$
\Gamma_{\mathcal{U}}(t, x):=\left\{u \in P(x) \mid\{-1\} \times f(x, u, v) \times\{-\mathbf{l}(x, u, v)\} \cap T_{\mathcal{U}}(t, x, y) \neq \emptyset\right\}
$$

Since $\mathcal{U}=\mathcal{E} p(\mathbf{u})$ since $\mathcal{U}$ is closed, it is the epigraph of an extended function denoted by $(t, x) \mapsto$ $\mathbf{u}(t, x)$.

When $y:=\mathbf{u}(t, x)$, the above condition (24) reads

$$
D_{\uparrow} \mathbf{u}(t, x)(-1, w) \leq-\mathbf{l}(x, u, v)
$$

because

$$
T_{\mathcal{U}}(t, x, \mathbf{u}(t, x))=\mathcal{E} p\left(D_{\uparrow} \mathbf{u}(t, x)\right)
$$

Conversely, this condition implies the tangential condition 24) for $y:=\mathbf{u}(t, x)$ whenever $(t, x, \mathbf{u}(t, x))$ belongs to $\mathcal{U}$. Otherwise, let $(t, x, y) \in \mathcal{U}$ with $y>\mathbf{u}(t, x)$ and set $\lambda:=D_{\uparrow} \mathbf{u}(t, x)(-1, w)$.

By definition of $\lambda:=D_{\uparrow} \mathbf{u}(t, x)(-1, w)$, there exist sequences $h_{n}>0$ converging to $0, w_{n}$ converging to $w$ and $\lambda_{n}$ converging to $\lambda$ such that $\left(t-h_{n}, x+h_{n} w_{n}, \mathbf{u}(t, x)+h_{n} \lambda_{n}\right)$ belongs to $\mathcal{E} p(\mathbf{u})$. Therefore, for $\mu \in \mathbf{R}$ and $h_{n}$ small enough,

$$
\left(t-h_{n}, x+h_{n} w_{n}, y+h_{n} \mu\right)=\left(t-h_{n}, x+h_{n} w_{n}, \mathbf{u}(t, x)+h_{n} \lambda_{n}\right)+\left(0,0, y-\mathbf{u}(t, x)+h_{n}\left(\mu-\lambda_{n}\right)\right)
$$

belongs to $\mathcal{E} p(\mathbf{u})$ because $y-\mathbf{u}(t, x)$ is strictly positive. This implies that $(-1, w, \mu)$ belongs to the contingent cone to the epigraph $\mathcal{U}$ of $\mathbf{u}$ at $(t, x, y)$, so that tangential condition (24) is satisfied with $\mu:=-\mathbf{l}(x, u, v)$. 


\section{The Auxiliary Guaranteed Capture Basin Under A Tychas- tic Control System}

\subsection{Introducing Auxiliary Tychastic Systems}

We observe that the evolution of $(T-t, x(t), p(t), y(t))$ made up of the backward time $\tau(t):=T-t$, of prices $x(t)$ of the shares, of portfolios $p(t)$ and of the capital $y(t)$ is governed by the tychastic system

$$
\begin{cases}(i) & \tau^{\prime}(t)=-1 \\ (\text { ii }) & \forall i=0, \ldots, n, \quad x_{i}^{\prime}(t)=x_{i}(t) \rho_{i}(x(t), v(t)) \\ (\text { iii }) & p^{\prime}(t)=u(t) \\ (i v) & y^{\prime}(t)=\sum_{i=0}^{n} p_{i}(t) x_{i}(t) \rho_{i}(x(t), v(t))+\langle u(t), x(t)\rangle \\ & \text { where } u(t) \in P(x(t), p(t)) \& v(t) \in Q(x(t))\end{cases}
$$

starting at $(T, x, p, y)$. We summarize it in the form of the tychastic system

$$
\begin{cases}(i) & z^{\prime}(t) \in g(z(t), u(t), v(t)) \\ (i i) & u(t) \in P(z(t)) \& v(t) \in Q(z(t))\end{cases}
$$

where $z:=(\tau, x, p, y) \in \mathbf{R} \times \mathbf{R}^{n+1} \times \mathbf{R}^{n+1} \times \mathbf{R}$, where the map $g: \mathbf{R} \times \mathbf{R}^{n+1} \times \mathbf{R}^{n+1} \times \mathbf{R} \leadsto$ $\mathbf{R} \times \mathbf{R}^{n+1} \times \mathbf{R}^{n+1} \times \mathbf{R}$ is defined by $g(z, u, v)$

$$
=\left(-1, x_{i}(t) \rho_{i}(x(t), v(t)), u, \sum_{i=0}^{n} p_{i}(t) x_{i}(t) \rho_{i}(x(t), v(t))+\langle u(t), x(t)\rangle\right)
$$

where $u$ ranges over $P(z):=P(t, x, p)$ and $v$ over $Q(z):=Q(t, x, p)$.

We say that a selection $z \mapsto \widetilde{u}(z) \in P(z)$ is a feedback, regarded as a strategy. One associates with such a feedback chosen by the manager or the player the evolutions governed by the perturbed differential equation

$$
z^{\prime}(t)=g(z(t), \widetilde{u}(z(t)), v(t))
$$

starting at time 0 at $z$.

\subsection{Introducing Guaranteed Capture Basins}

We now define the guaranteed viable-capture basin that is involved in the definition of guaranteed valuation subsets.

Definition 3.1 Let $K$ and $C \subset K$ be two subsets of $Z$.

The guaranteed viable-capture basin of the target $C$ viable in $K$ is the set of elements $z \in K$ such that there exists a continuous feedback $\widetilde{u}(z) \in P(z)$ such that for every $v(\cdot) \in Q(z(\cdot))$, for every solutions $z(\cdot)$ to $z^{\prime}=g(z, \widetilde{u}(z), v)$, there exists $t^{*} \in \mathbf{R}_{+}$such that the viability/capturability conditions

$$
\left\{\begin{array}{lll}
(i) & \forall t \in\left[0, t^{*}\right], & z(t) \in K \\
(i i) & z\left(t^{*}\right) \in C
\end{array}\right.
$$

are satisfied. 
We thus observe that

Proposition 3.2 The guaranteed valuation subset $\mathcal{V}_{(\mathbf{b}, \mathbf{c})}^{\sharp}$ defined in Definition 1.4 is related to the guaranteed viable-capture basin under the tychastic system (25) of the epigraph of the objective function c viable in the epigraph of the constraint function $\mathbf{b}$.

The characterization of this subset and the study of its properties are one of the major topics of the viability approach to dynamical games theory that we summarize in the two next sections.

\subsection{Analytical Formula of the Valuation Function}

We set $\mathbf{l}(x, p, u, v):=-\sum_{i=0}^{n} p_{i} x_{i} \rho_{i}(x, v)-\langle u, x\rangle$ and we obtain

$$
\left\{\begin{array}{l}
J_{\mathbf{c}}(t ;(x(\cdot), p(\cdot), v(\cdot)) ; \widetilde{u})(T, x, p):=\mathbf{c}(T-t, x(t), p(t)) \\
\left.\left.+\int_{0}^{t}\left(\sum_{i=0}^{n} p_{i}(\tau) x_{i}(\tau) \rho_{i}\left(x_{i}(\tau), v(\tau)\right)-\langle\widetilde{u}(T-\tau, x(\tau), p(\tau)), v(\tau))\right), x(\tau)\right\rangle\right) d \tau
\end{array}\right.
$$

We shall associate with it three pairs of time-dependent functions $(\mathbf{b}, \mathbf{c})$ and obtain three different valuation functions of portfolios duplicating European, American options and first time options:

1. European case: We take $\mathbf{b}(t, x, p):=0$ and $\mathbf{c}(t, x, p):=\mathbf{u}_{\infty}(t, x, p)$. In this case, we obtain

$$
V_{\left(\mathbf{0}, \mathbf{u}_{\infty}\right)}^{\sharp}(T, x, p):=\inf _{\widetilde{u}(t, x, p) \in P(t, x, p)(x(\cdot), p(\cdot), v(\cdot)) \in \mathcal{C}_{\tilde{u}}(x, p)} \sup _{\mathbf{u}}(T ;(x(\cdot), p(\cdot), v(\cdot)) ; \widetilde{u})(x, p)
$$

2. American case: We take $\mathbf{b}(t, x, p):=\mathbf{u}(x, p)$ and $\mathbf{c}(t, x, p):=\mathbf{u}_{\infty}(t, x, p)$. In this case, we obtain

$$
V_{\left(\mathbf{u}, \mathbf{u}_{\infty}\right)}^{\sharp}(T, x, p):=\inf _{\widetilde{u}(x) \in P(x, p)} \sup _{(x(\cdot), p(\cdot), v(\cdot)) \in \mathcal{C}_{\widetilde{u}}(x, p)} \sup _{t \in[0, T]} J_{\mathbf{u}}(t ;(x(\cdot), p(\cdot), v(\cdot)) ; \widetilde{u})(x, p)
$$

3. Kairotic case: We assume that $\mathbf{l} \geq 0$ and we take $\mathbf{b}(t, x, p):=0$ and $\mathbf{c}(t, x, p)=\mathbf{u}(x, p)$. In this case, we obtain

$$
V_{(\mathbf{0}, \mathbf{u})}^{\sharp}(T, x, p):=\inf _{\widetilde{u}(x) \in P(x, p)} \sup _{(x(\cdot), p(\cdot), v(\cdot)) \in \mathcal{C}_{\widetilde{u}}(x, p)} \inf _{t \in[0, T]} J_{\mathbf{u}}(t ;(x(\cdot), p(\cdot), v(\cdot)) ; \widetilde{u})(x, p)
$$

We have to compute the functionals $J_{\mathbf{u}}, J_{\mathbf{u}_{\infty}}, \mathbf{I}_{\mathbf{0}}$ and $\mathbf{I}_{\mathbf{u}}$ to obtain our three formulas.

First, we observe that for $\mathbf{c}(t, x, p):=\mathbf{u}(x, p)$,

$$
\left\{\begin{array}{l}
J_{\mathbf{u}}(t ;(x(\cdot), p(\cdot), v(\cdot)) ; \widetilde{u})(T, x, p):=\mathbf{u}(x(t), p(t)) \\
\left.\left.+\int_{0}^{t}\left(\sum_{i=0}^{n} p_{i}(\tau) x_{i}(\tau) \rho_{i}\left(x_{i}(\tau), v(\tau)\right)-\langle\widetilde{u}(T-\tau, x(\tau), p(\tau)), v(\tau))\right), x(\tau)\right\rangle\right) d \tau
\end{array}\right.
$$

Second, when we take $\mathbf{c}(t, x, p):=\mathbf{u}_{\infty}(t, x, p)$ that takes infinite values for $t>0$, we observe that

$$
J_{\mathbf{u}_{\infty}}(t ;(x(\cdot), p(\cdot), v(\cdot)) ; \widetilde{u})(T, x, p):= \begin{cases}J_{\mathbf{u}}(T ;(x(\cdot), p(\cdot), v(\cdot)) ; \widetilde{u})(T, x, p) & \text { if } \quad t=T \\ +\infty & \text { if } \quad t \in[0, T[\end{cases}
$$


so that

$$
\inf _{t \in[0, T]} J_{\mathbf{u}_{\infty}}(t ;(x(\cdot), p(\cdot), v(\cdot)) ; \widetilde{u})(T, x, p):=J_{\mathbf{u}}(T ;(x(\cdot), p(\cdot), v(\cdot)) ; \widetilde{u})(T, x, p)
$$

Third, when $\mathbf{b}=0$, we observe that

$$
J_{\mathbf{0}}(t ;(x(\cdot), p(\cdot), v(\cdot)) ; \widetilde{u})(T, x, p)=\int_{0}^{t} \mathbf{l}(x(\tau), p(\tau), \widetilde{u}(x(\tau), p(\tau)), v(\tau)) d \tau
$$

so that,

$$
\left\{\begin{array}{l}
I_{\mathbf{0}}(t ;(x(\cdot), p(\cdot), v(\cdot)) ; \widetilde{u})(T, x, p) \\
=\sup _{s \in[0, t]} \int_{0}^{s} \mathbf{l}(x(\tau), p(\tau), \widetilde{u}(x(\tau), p(\tau)), v(\tau)) d \tau
\end{array}\right.
$$

Finally, when $\mathbf{b}(t, x, p)=\mathbf{u}(x, p)$, we observe that

$$
\left\{\begin{array}{l}
I_{\mathbf{u}}(t ;(x(\cdot), p(\cdot), v(\cdot)) ; \widetilde{u})(T, x, p) \\
=\sup _{s \in[0, t]}\left(\mathbf{u}(x(t), p(t))+\int_{0}^{s} \mathbf{l}(x(\tau), p(\tau), \widetilde{u}(T-\tau, x(\tau), p(\tau)), v(\tau)) d \tau\right)
\end{array}\right.
$$

\subsection{Black and Scholes type Variational Inequalities}

Let us associate with a nonnegative extended function $\mathbf{v}$ the subset

$$
\begin{gathered}
\Omega_{(\mathbf{b}, \mathbf{c})}(\mathbf{v}):=\left\{(t, x, p) \in \mathbf{R}_{+} \times \mathbf{R}^{n+1} \times \mathbf{R}^{n+1}\right. \text { such that } \\
\mathbf{b}(t, x, p) \leq \mathbf{v}(t, x, p)<\mathbf{c}(t, x, p)\}
\end{gathered}
$$

which depends of the function $\mathbf{v}$.

Example When for all $t>0, \mathbf{c}(t, x, p):=+\infty$, and when $\mathbf{b}(0, x, p):=\mathbf{c}(0, x, p)$, we observe that

$$
\begin{gathered}
\Omega_{(\mathbf{b}, \mathbf{c})}(\mathbf{v}):=\left\{(t, x, p) \in \mathbf{R}_{+} \times \mathbf{R}^{n+1} \times \mathbf{R}^{n+1} \quad\right. \text { such that } \\
\\
t>0 \& \mathbf{b}(t, x, p) \leq \mathbf{v}(t, x, p)\}
\end{gathered}
$$

We shall prove that the guaranteed value-function $V_{(\mathbf{b}, \mathbf{c})}^{\sharp}$ is a "contingent" solution $\mathbf{v}$ to the Hamilton-Jacobi-Isaacs variational inequality: for every $(t, x, p) \in \Omega_{(\mathbf{b}, \mathbf{c})}(\mathbf{v})$,

$$
\left\{\begin{array}{l}
\frac{\partial \mathbf{v}(t, x, p)}{\partial t}=\inf _{u \in P(x, p)}\left(\sum_{i=0}^{n} \frac{\partial \mathbf{v}(t, x, p)}{\partial p_{i}} u_{i}\right. \\
\left.+\sup _{v \in Q(x)}\left(\sum_{i=0}^{n} \frac{\partial \mathbf{v}(t, x, p)}{\partial x_{i}} x_{i} \rho_{i}(x, v)+\mathbf{l}(x, p, u, v)\right)\right)
\end{array}\right.
$$

This is a free boundary problem, well studied in mechanics and physics: the domain $\Omega_{(\mathbf{b}, \mathbf{c})}(\mathbf{v})$ on which we look for a solution $\mathbf{v}$ to the Hamilton-Jacobi partial differential equation depends upon the unknown solution $\mathbf{v}$.

Observe that Hamilton-Jacobi partial differential equation itself depends only upon the dynamic of the system $(\rho, P, Q)$ and the map $\mathbf{l}$, whereas the domain $\Omega_{(\mathbf{b}, \mathbf{c})}(\mathbf{v})$ depends only upon the pair $(\mathbf{b}, \mathbf{c})$ 
describing the dynamical constraints and the objective. Changing them, the valuation function is a solution of the same Hamilton-Jacobi partial differential equation, but defined on different "free sets" $\Omega_{(\mathbf{b}, \mathbf{c})}(\mathbf{v})$ depending on $\mathbf{v}$.

The usefulness and relevance of the Hamilton-Jacobi-Isaacs variational inequality is that it provides the adjustment law - through dynamical feedbacks - that we are looking for. Indeed, we introduce the regulation map $\Gamma$ associating with any $(t, x, p) \in \mathbf{R}_{+} \times \mathbf{R}^{n+1} \times \mathbf{R}^{n+1}$ the subset $\Gamma(t, x, p)$ of strategies $u \in P(x, p)$ satisfying

$$
\left\{\begin{array}{l}
\sum_{i=0}^{n} \frac{\partial V_{(\mathbf{b}, \mathbf{c})}^{\sharp}(t, x, p)}{\partial p_{i}} u_{i} \\
+\sup _{v \in Q(x)}\left(\sum_{i=0}^{n} \frac{\partial V_{(\mathbf{b}, \mathbf{c})}^{\sharp}(t, x, p)}{\partial x_{i}} x_{i} \rho_{i}(x, v)+\mathbf{l}(x, p, u, v)\right) \\
\leq \frac{\partial V_{(\mathbf{b}, \mathbf{c})}^{\sharp}(t, x, p)}{\partial t}
\end{array}\right.
$$

The adjustment law states in essence that the evolution of the portfolio allowing to satisfy the viability/capturability conditions (3) against all perturbations $v \in Q(t, x, p)$ is governed by the differential inclusion

$$
p^{\prime}(t) \in \Gamma(T-t, x(t), p(t))
$$

Namely, knowing the guaranteed valuation function and its derivatives, a guaranteed solution is obtained in the following way: Starting from $x_{0}$ and $p_{0}$ such that $\mathbf{b}\left(T, x_{0}, p_{0}\right) \leq V_{(\mathbf{b}, \mathbf{c})}^{\sharp}\left(T, x_{0}, p_{0}\right)<$ $\mathbf{c}\left(T, x_{0}, p_{0}\right)$, solutions to the new system

$$
\begin{cases}(i) & \forall i=0, \ldots, n, x_{i}^{\prime}(t)=x_{i}(t) \rho_{i}(x(t), v(t)) \\ (i i) & p^{\prime}(t) \in \Gamma(T-t, x(t), p(t)) \\ (i i i) & y^{\prime}(t)=-\mathbf{l}(x(t), p(t), u(t), v(t))\end{cases}
$$

regulate the guaranteed solutions of the tychastic system until the first time $t^{*} \in[0, T]$ when

$$
V_{(\mathbf{b}, \mathbf{c})}^{\sharp}\left(T-t^{*}, x\left(t^{*}\right), p\left(t^{*}\right)\right)=\mathbf{c}\left(T-t^{*}, x\left(t^{*}\right), p\left(t^{*}\right)\right)
$$

Setting $\mathbf{l}(x, p, u, v):=-\sum_{i=0}^{n} p_{i} x_{i} \rho_{i}(x, v)-\langle u, x\rangle$, the Hamilton-Jacobi-Isaacs variational inequality becomes : for every $(t, x, p) \in \Omega_{(\mathbf{b}, \mathbf{c})}(\mathbf{v})$,

$$
-\frac{\partial V^{\sharp}(t, x, p)}{\partial t}+\inf _{u \in P(x, p)} \sum_{i=0}^{n}\left(\frac{\partial V^{\sharp}(t, x, p)}{\partial p_{i}}-x_{i}\right) u_{i}+\sup _{v \in Q(x)}\left(\sum_{i=0}^{n}\left(\frac{\partial V^{\sharp}(t, x, p)}{\partial x_{i}}-p_{i}\right) x_{i} \rho_{i}(x, v)\right)=0
$$

and the regulation map $\Gamma$ associates with any $(T, x, p) \in \mathbf{R}_{+} \times \mathbf{R}^{n+1} \times \mathbf{R}^{n+1}$ the subset $\Gamma(T, x, p)$ of $u \in P(x, p)$ satisfying

$$
\left\{\begin{array}{l}
\sum_{i=0}^{n}\left(\frac{\partial V^{\sharp}(t, x, p)}{\partial p_{i}}-x_{i}\right) u_{i}+\sup _{v \in Q(x)}\left(\sum_{i=0}^{n}\left(\frac{\partial V^{\sharp}(t, x, p)}{\partial x_{i}}-p_{i}\right) x_{i} \rho_{i}(x, v)\right) \\
\leq \frac{\partial V^{\sharp}(t, x, p)}{\partial t}
\end{array}\right.
$$




\section{References}

[1] AUBIN J.-P. (1981) Contingent derivatives of set-valued maps and existence of solutions to nonlinear inclusions and differential inclusions, Advances in Mathematics, Supplementary studies, Ed. Nachbin L., 160-232

[2] AUBIN J.-P. (1986) A viability approach to Lyapunov's second method, In DynAmicAl Systems, Kurzhanski A. and Sigmund K., Lectures Notes in Economics and Math. Systems, Springer-Verlag, $287,31-38$

[3] AUBIN J.-P. (1991) Viability Theory Birkhäuser, Boston, Basel, Berlin

[4] AUBIN J.-P. (1997) Dynamic Economic Theory: A Viability Approach, Springer-Verlag

[5] AUBIN J.-P. (1999) Impulse Differential Inclusions and Hybrid Systems: A Viability Approach, Lecture Notes, University of California at Berkeley

[6] AUBIN J.-P. (2000) Lyapunov Functions for Impulse and Hybrid Control Systems, Proceedings of the CDC 2000 Conference

[7] AUBIN J.-P. (2000) Optimal Impulse Control Problems and Quasi-Variational Inequalities Thirty Years Later: a Viability Approach, in Contrôle optimal et EDP: Innovations et Applications, IOS Press

[8] AUBIN J.-P., BAYEN A., BONNEUIL N. \& SAINT-PIERRE P. (in preparation) Viability, Control and Games: Regulation of Complex Evolutionary Systems Under Uncertainty and Viability Constraints

[9] AUBIN J.-P. \& CATTE F. (2001) Fixed-Point and Algebraic Properties of Viability Kernels and Capture Basins of Sets,

[10] AUBIN J.-P. \& CELLINA A. (1984) Differential Inclusions, Springer-Verlag

[11] AUBIN J.-P. \& DA PRATO G. (1995) Stochastic Nagumo's Viability Theorem, Stochastic Analysis and Applications, 13, 1-11

[12] AUBIN J.-P. \& DA PRATO G. (1998) The Viability Theorem for Stochastic Differential Inclusions, Stochastic Analysis and Applications, 16, 1-15

[13] AUBIN J.-P., DA PRATO \& FRANKOWSKA (2000) Stochastic Invariance for Differential Inclusions, Set-Valued Analysis

[14] AUBIN J.-P. \& DORDAN O. (1996) Fuzzy Systems, Viability Theory and Toll Sets, In Handbook of Fuzzy Systems, Modeling and Control, Hung Nguyen Ed.. Kluwer, 461-488

[15] AUBIN J.-P. \& DOSS H. (2001) Itô and Stratonovitch Stochastic Viability,

[16] AUBIN J.-P. \& FRANKOWSKA H. (1990) Set-Valued Analysis, Birkhäuser

[17] AUBIN J.-P. \& FRANKOWSKA H. (1996) The Viability Kernel Algorithm for Computing Value Functions of Infinite Horizon Optimal Control Problems, J.Math. Anal. Appl., 201, 555-576

[18] AUBIN J.-P. \& HADDAD G. (2001) Cadenced runs of impulse and hybrid control systems, International Journal Robust and Nonlinear Control 
[19] AUBIN J.-P. \& HADDAD G. (2001) Path-Dependent Impulse and Hybrid Systems, in Hybrid Systems: Computation and Control, 119-132, Di Benedetto \& Sangiovanni-Vincentelli Eds, Proceedings of the HSCC 2001 Conference, LNCS 2034, Springer-Verlag

[20] AUBIN J.-P. \& HADDAD G. (2002) History (Path) Dependent Optimal Control and Portfolio Valuation and Management, J. Positivity

[21] AUBIN J.-P., LYGEROS J., QUINCAMPOIX. M., SASTRY S. \& SEUBE N. (to appear) Impulse Differential Inclusions: A Viability Approach to Hybrid Systems,

[22] BARDI M. \& CAPUZZO DOLCETTA I. (1998) Optimal control and viscosity solutions to Hamilton-Jacobi-Bellman equations, Birkhäuser

[23] BARDI M. \& GOATIN P. (1997) A Dirichlet type problem for nonlinear degenerate elliptic equations arinsing in time-optimal stochastic control, Preprint SISSA 50/97

[24] BARDI M. \& GOATIN P. (1998) Invariant sets for controlled degenerate diffusions: a viscosity solution approach, Preprint Padova 2/98

[25] BENSOUSSAN A. \& LIONS J.-L. (1982) Contrôle impulsionnel et inéquations quasivariationnelles, Dunod, Paris (Eblish translation: (1984) Impulse control ad quasivariational inequalities, Gauthier-Villars)

[26] BEnSOUSSAN A. \& MENALDI (1997) Hybrid Control and Dynamic Programming, Dynamics of Continuous, Discrete and Impulse Systems, 3, 395-442

[27] BERNHARD P. (2000) Max-Plus algebra and mathematical fear in dynamic optimization, J. SetValued Analysis,

[28] BERNHARD P. (2000) Une approche déterministe de l'évaluation des options, in Optimal CONtrol and Partial Differential Equations, IOS Press

[29] BERNHARD P. (2000) A robust control approach to option pricing, Cambridge University Press

[30] BERNHARD P. (2002) Robust control approach to option pricing, including transaction costs, Annals of Dynamic Games

[31] BJORK T. (1998) Arbitrage theory in continuous time, Oxford University Press

[32] BUCKDAHN R., QUINCAMPOIX M. \& RASCANU A. h (1997) Propriétés de viabilité pour des équations différentielles stochastiques rétrogrades et applications à des équations aux dérivées partielles, Comptes-Rendus de l'Académie des Sciences, 235, 1159-1162

[33] BUCKDAHN R., QUINCAMPOIX M. \& RASCANU A. (1998) Stochastic viability for backward stochastic

differential equations and applications to partial differential equations, Un. Bretagne Occidentale, 01-1998

[34] BUCKDAHN R., PENG S., QUINCAMPOIX M. \& RAINER C. (1998) Existence of stochastic control under state constraints, Comptes-Rendus de l'Académie des Sciences, 327, 17-22

[35] BUCKDAHN R., CARDALIAGUET P. \& QUINCAMPOIX M. (2000) A representation formula for the mean curvature motion, UBO 08-2000 
[36] CARDAliaguet P. (1994) Domaines dicriminants en jeux différentiels, Thèse de l'Université de Paris-Dauphine

[37] CARDALIAGUET P. (1996) A differential game with two players and one target, SIAM J. Control and Optimization, 34, 4, 1441-1460.

[38] CARDALIAGUET P. (2000) Introduction à la théorie des jeux différentiels, Lecture Notes, Université Paris-Dauphine

[39] CARDAliagUET P., QUINCAMPOIX M. \& SAINT-PIERRE P. (1999) Set-valued numerical methods for optimal control and differential games, In Stochastic and differential games. Theory and numerical methods, Annals of the International Society of Dynamical Games, 177-247 Birkhäuser

[40] COX J. \& RUBinstein M. (1985) Options Market, Prentice Hall.

[41] DA PRATO \& FRANKOWSKA (1994) A stochastic Filippov Theorem, Stochastic Calculus, 12, 409-426

[42] DA PRATO \& FRANKOWSKA (2001) Stochastic viability for compact sets in terms of the distance function, Dynamics Systems Appl., 10, 177-184

[43] DA PRATO \& FRANKOWSKA (2004) Invariance of stochastic control systems with deterministic arguments, J. Differential Equations, 200. 18-52

[44] DOSS H. (1977) Liens entre équations différentielles stochastiques et ordinaires, Ann. Inst. henri Poincaré, Calcul des Probabilités et Statistique, 23, 99-125

[45] FILIPOVIC D. (1999) Invariant manifolds for weak solutions to stochastic equations, preprint

[46] FRANKOWSKA H. (1989) Optimal trajectories associated to a solution of contingent HamiltonJacobi equations, Applied Mathematics and Optimization, 19, 291-311

[47] FRANKOWSKA H. (1989) Hamilton-Jacobi equation: viscosity solutions and generalized gradients, J. of Math. Analysis and Appl. 141, 21-26

[48] FRANKOWSKA H. (1991) Lower semicontinuous solutions to Hamilton-Jacobi-Bellman equations, Proceedings of 30th CDC Conference, IEEE, Brighton, December 11-13

[49] FRANKOWSKA H. (1993) Lower semicontinuous solutions of Hamilton-Jacobi-Bellman equation, SIAM J. on Control and Optimization,

[50] GAUTIER S. \& THIBAULT L. (1993) Viability for constrained stochastic differential equations, Differential Integral Equations, 6, 1395-1414

[51] HADDAD G. (1981) Monotone trajectories of differential inclusions with memory, Isr. J. Math., 39, 83-100

[52] HADDAD G. (1981) Monotone viable trajectories for functional differential inclusions, J. Diff. Eq., 42, 1-24

[53] HADDAD G. (1981) Topological properties of the set of solutions for functional differential differential inclusions, Nonlinear Anal. Theory, Meth. Appl., 5, 1349-1366

[54] JACHIMIAK W. (1996) A note on invariance for semilinear differential equations, Bull. Pol. Acad. Sc., 44, 179-183 
[55] JACHIMIAK W. (1998) Stochastic invariance in infinite dimension, preprint

[56] KISIELEWICZ M. (1995) Viability theorem for stochastic inclusions, Discussiones Mathematicae, Differential Inclusions, 15, 61-74

[57] MILIAN A. (1995) Stocastic viability and a comparison theorem, Colloq. Math.,68,297-316

[58] MILIAN A. (1997) Invariance for stochastic equations with regular coefficients, Stochastic Anal. Appl., 15, 91-101

[59] MILIAN A. (1998) Comparison theorems for stochastic evolution equations, preprint

[60] OLSDER G.J. (1999) Control-theoretic thoughts on option pricing, preprint

[61] PUJAL D. (2000) Valuation et gestion dynamiques de portefeuilles, Thèse de l'Université de ParisDauphine

[62] PUJAL D. \& SAINT-PIERRE P. (2001) L'algorithme du bassin de capture appliqué pour évaluer des options européennes, américaines ou exotiques, preprint

[63] QUINCAMPOIX M. (1992) Differential inclusions and target problems, SIAM J. Control and Optimization, 30, 324-335

[64] ROCKAFELLAR R.T. \& WETS R. (1997) Variational Analysis, Springer-Verlag

[65] RUNGGALDIER W.J. (2000) Adaptive and robust control peocedures for risk minimization under uncertainty, in Optimal Control and Partial Differential Equations, 511-520, IOS Press

[66] SAINT-PIERRE P. (1994) Approximation of the viability kernel, Applied Mathematics \& Optimisation, 29, 187-209

[67] SAINT-PIERRE P. (to appear) Approximation of capture basins for hybrid systems,

[68] SONER H.M., SHREVE S.E. \& CVITANIC J. (1995) There is no trivial hedging for option pricing with transaction costs, The Annals of Probability, 5, 327-355

[69] SONER H.M. \& TOUZI N. (1998) Super-replication under Gamma constraints, SIAM J. Control and Opt., 39, 73-96

[70] SONER H.M. \& TOUZI N. (2000) Dynamic programming for a class of control problems,

[71] SONER H.M. \& TOUZI N. (to appear) Stochastic target problems, dynamical programming and viscosity solutions,

[72] TESSITORE G. \& ZABCZYK J. (1998) Comments on transition semi-groups and stochastic invariance, preprint

[73] ZABCZYK J. (1973) Optimal control by means of switching, Studia Matematica, 65, 161-171

[74] ZABCZYK J. (1992) Mathematical control theory: An introduction, Birkhäuser

[75] ZABCZYK J. (1996) Chance and decision: stochastic control in discrete time, Quaderni, Scuola Normale di Pisa

[76] ZABCZYK J. (1999) Stochastic invariance and consistency of financial models, preprint Scuola Normale di Pisa 


\section{Contents}

$1 \quad$ Introduction and Survey of the Main Results 2

1.1 Statement of the Problems . . . . . . . . . . . . . . . . . . . . . . . . 2

1.2 Underlying Viability/Capturability Problem . . . . . . . . . . . . . . . . . . . . . . . . 3

1.3 The Dynamics . . . . . . . . . . . . . . . . . . . . . . . . . . . . 6

1.4 Dynamic Management and Valuation of the Portfolio . . . . . . . . . . . . . . . . . . . 9

1.5 A Numerical Example . . . . . . . . . . . . . . . . . . . . . . . . . 10

1.6 Hamilton-Jacobi Inequalities and Regulation Maps . . . . . . . . . . . . . . . . . . . . 14

1.7 Analytical Formulas for the Valuation Functions . . . . . . . . . . . . . . . . . . . . . 15

\begin{tabular}{|lll}
2 & The Viability/Capturability Strategy & 16
\end{tabular}

2.1 Absorption Basins under Tychastic systems . . . . . . . . . . . . . . . . . . . . . . . 18

2.2 Guaranteed Viable-Capture Basins . . . . . . . . . . . . . . . . . . . . . . . . . . . . . 21

2.3 Intertemporal Games . . . . . . . . . . . . . . . . . . . . . . . . . . . . . . . . . 23

2.4 Hamilton-Jacobi-Isaacs Variational Inequalities . . . . . . . . . . . . . . . . . . . . . . 24

3 The Auxiliary Guaranteed Capture Basin Under A Tychastic Control System 26

3.1 Introducing Auxiliary Tychastic Systems . . . . . . . . . . . . . . . . . . . . . . . 26

3.2 Introducing Guaranteed Capture Basins . . . . . . . . . . . . . . . . . . . . . . . . . . 26

3.3 Analytical Formula of the Valuation Function . . . . . . . . . . . . . . . . . . . . . . . 27

3.4 Black and Scholes type Variational Inequalities . . . . . . . . . . . . . . . . . . . . . . 28 